ITFA-2006-49

hep-ph/0612089

\title{
Unitarity Cuts with Massive Propagators and Algebraic Expressions for Coefficients
}

\author{
Ruth Britto $^{a}$ and Bo Feng ${ }^{b, c}$ \\ ${ }^{a}$ Institute for Theoretical Physics, University of Amsterdam \\ Valckenierstraat 65, 1018 XE Amsterdam, The Netherlands \\ ${ }^{b}$ Center of Mathematical Science, Zhejiang University, Hangzhou, China \\ ${ }^{c}$ Blackett Laboratory 8 The Institute for Mathematical Sciences \\ Imperial College, London, SW7 2AZ, UK
}

\begin{abstract}
In the first part of this paper, we extend the $d$-dimensional unitarity cut method of hepph/0609191 to cases with massive propagators. We present formulas for integral reduction with which one can obtain coefficients of all pentagon, box, triangle and massive bubble integrals. In the second part of this paper, we present a detailed study of the phase space integration for unitarity cuts. We carry out spinor integration in generality and give algebraic expressions for coefficients, intended for automated evaluation.
\end{abstract}

KeYWORDS: . 


\section{Contents}

1. Introduction 1

2. Unitarity cuts with massive propagators 3

2.1 Cuts of Scalar Integrals 3

2.2 Kinematics and the domain of integration 5

2.3 First steps: separating the four-dimensional integral 6

3. Recursion and reduction formulas with mass 6

3.1 Bubble

3.2 Triangle 8

3.3 Box 10

3.4 Pentagon 12

4. Formulas for coefficients from double cuts 13

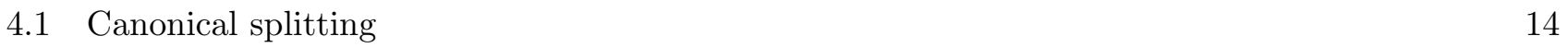

4.2 Box and triangle coefficients 16

\begin{tabular}{ll}
4.3 & Rational part \\
\hline
\end{tabular}

\begin{tabular}{ll}
4.4 & Summary of results \\
\hline
\end{tabular}

5. Discussion 2

\begin{tabular}{llr}
\hline 5.1 & Tree level input & 24 \\
\hline
\end{tabular}

\begin{tabular}{lll}
5.2 & The quadruple cut & 25 \\
\hline
\end{tabular}

\begin{tabular}{llr}
5.3 & Comparison with OPP method & 26 \\
\hline
\end{tabular}

A. Evaluation of residues $\quad 27$

\begin{tabular}{lll}
\hline A.1 Box coefficients & 27 \\
\hline
\end{tabular}

\begin{tabular}{lll}
\hline A.2 The residue of multiple poles & 28
\end{tabular}

\section{Introduction}

With the approach of the Large Hadron Collider experiments, accurate descriptions of particle physics will require knowledge of one-loop cross sections. Computational complexity increases dramatically with 
the number of legs, even at the amplitude level. It is desirable to find a simple and fast algorithm for these computations. There has been notable progress in the last couple of years [1, 2, 3, 4, 5, 6, 0, 8, 9, 10, 11].

The unitarity method introduced in [12] seeks to compute amplitudes by applying a unitarity cut to an amplitude on one hand, and its expansion in a basis of master integrals on the other [13. With knowledge of the basis and the general structure of the coefficients in the expansion, the coefficients can be constrained.

The holomorphic anomaly [14] reduces the problem of phase space integration to one of algebraic manipulation, namely evaluating residues of a complex function. By applying this operation within the unitarity method, coefficients can be extracted systematically. In this manner, a method was introduced to evaluate any finite four-dimensional unitarity cut and systematically derive compact expressions for the coefficients [6, 7].

When working with four-dimensional cuts, one loses information of possible rational terms that are cut-free in four dimensions. However, unitarity methods can find rational terms as well, if we carry out the integral in $d=(4-2 \epsilon)$ dimensions to higher orders in $\epsilon$ [15]. This program was developed in [16, 17, 18, 9].

Recently, a general $d$-dimensional unitarity cut method was developed for one-loop amplitudes [19, 20]. In this method, coefficients are extracted by first separating and performing a four-dimensional integral by a technique of choice, and then identifying the integral over the remaining $d-4$ dimensions with a coefficient using recursive dimensional shift identities. In principle one can work out the coefficients to all orders in $\epsilon$. In fact, for cases with only massless propagators, this method is a complete alternative to Passarino-Veltman reduction.

In the first part of this paper (Sections 2 and 3) we generalize the work of [19, 20] to cases where propagators have nonzero, non-uniform masses. We present formulas for integral reduction from which one can obtain coefficients of all scalar pentagon, box, triangle and massive bubble integrals. Pentagons in arbitrary dimensions are truly independent master integrals, though in four dimensions they can be expressed in terms of boxes up to $\mathcal{O}(\epsilon)$. Amplitudes allowing massive propagators will most generally have tadpole and massless-bubble master integrals as well. Unitarity methods cannot access this information directly, but other physical considerations pin it down.

In the second part (Section 4), we further simplify the technique of spinor integration over phase space by carrying out some intermediate steps in generality. These steps include expressing the integrand as a total derivative and a possible Feynman-parameter integral. We give algebraic expressions for the coefficients of the cut-containing basis integrals. Our formulas should be suitable for programming. To this end we include some possibly helpful identities in the appendix, as well as a discussion of the issue of multiple poles.

We close with a discussion (Section 5) of the tree-level input and possible alternative approaches. We compare our approach of spinor integration to the procedure of Ossola, Papadopoulos and Pittau [2] of evaluating integrands at particular values of loop momentum and solving a system of linear equations. Both our method and theirs algebraic substitution at the integrand level. We claim that the complexity is 
equivalent.

\section{Unitarity cuts with massive propagators}

Here we develop the program of double-cut phase space integration in $(4-2 \epsilon)$ dimensions 16, 17, 18, 9, 19, 20] by generalizing to the case of propagators with nonzero and different masses.

One difference from the massless case is in the basis of master integrals. If all propagators are massless, the basis consists of scalar pentagons, boxes, triangles and bubbles. But if some propagators are massive, there are tadpoles and massless bubbles (i.e., the case $K^{2}=0$ for bubbles). The tadpoles are [16]

$$
\begin{aligned}
I_{1} & \sim m^{2-2 \epsilon} \frac{\Gamma[1+\epsilon]}{\epsilon(\epsilon-1)} \\
& =\frac{m^{2}}{\epsilon}+m^{2}(1-\gamma-2 \log (m))+\mathcal{O}(\epsilon),
\end{aligned}
$$

while for massless bubbles we find

$$
\begin{aligned}
I_{2}(K=0) & \sim \frac{M_{1}^{2-2 \epsilon}-M_{2}^{2-2 \epsilon}}{M_{1}^{2}-M_{2}^{2}} \frac{\Gamma[1+\epsilon]}{\epsilon(1-\epsilon)} \\
& =\frac{1}{\epsilon}+(1-\gamma)-\frac{2}{M_{1}^{2}-M_{2}^{2}}\left(M_{1}^{2} \log \left(M_{1}\right)-M_{2}^{2} \log \left(M_{2}\right)\right)+\mathcal{O}(\epsilon) \\
& \text { or } \frac{1}{\epsilon}-\left(\gamma+2 \log M_{1}\right)+\mathcal{O}(\epsilon) \quad \text { if } \quad M_{1}=M_{2} .
\end{aligned}
$$

It is evident from the lack of all momentum dependence that unitarity methods alone can never detect these two functions.

However, as discussed in [16], it is possible in some cases to address this difficulty by considering the known divergent behavior of the amplitude. For massless gauge theory amplitudes, the conditions are such that quadratic divergences cancel and the remaining (logarithmic) divergence matches the known value. With these conditions we can fix coefficients at leading order in $\epsilon$. In more general cases, we might need some additional information. This could come, for example, from taking the heavy mass limit of one propagator [21]. We shall not discuss the tadpoles and massless bubbles further in this paper.

\subsection{Cuts of Scalar Integrals}

We define the $n$-point scalar function with non-uniform masses as follows: ${ }^{1}$

$$
I_{n}\left(M_{1}, M_{2}, m_{1}, \ldots, m_{n-2}\right) \equiv \int \frac{d^{4-2 \epsilon} p}{(2 \pi)^{4-2 \epsilon}} \frac{1}{\left(p^{2}-M_{1}^{2}\right)\left((p-K)^{2}-M_{2}^{2}\right) \prod_{j=1}^{n-2}\left(\left(p-P_{j}\right)^{2}-m_{j}^{2}\right)} .
$$

This definition is written with a view towards taking the unitarity cut. The masses of the cut propagators are $M_{1}$ and $M_{2}$, and the momentum flowing through the cut is $K$. The other momenta $P_{j}$ and masses $m_{j}$ are the ones necessary to complete the scalar function, and not necessarily in cyclic order.

\footnotetext{
${ }^{1}$ Note that, for ease of presentation, we are omitting the prefactor $i(-1)^{n+1}(4 \pi)^{D / 2}$ (which was included for example in [16]).
} 
Our calculations are done in the "Four Dimensional Helicity" (FDH) scheme, i.e. all external momenta $K_{i}$ are 4-dimensional and only the internal momentum $p$ is $(4-2 \epsilon)$-dimensional. Thus it is useful to write $p=\tilde{\ell}+\vec{\mu}$, where $\tilde{\ell}$ is 4 -dimensional and $\vec{\mu}$ is $(-2 \epsilon)$-dimensional.

$$
\int \frac{d^{4-2 \epsilon} p}{(2 \pi)^{4-2 \epsilon}}=\int \frac{d^{4} \widetilde{\ell}}{(2 \pi)^{4}} \int \frac{d^{-2 \epsilon} \ell_{\epsilon}}{(2 \pi)^{-2 \epsilon}}=\int \frac{d^{4} \tilde{\ell}}{(2 \pi)^{4}} \frac{(4 \pi)^{\epsilon}}{\Gamma(-\epsilon)} \int d \mu^{2}\left(\mu^{2}\right)^{-1-\epsilon} .
$$

Then the scalar function as defined in (2.5) becomes

$$
\begin{aligned}
I_{n}\left(M_{1}, M_{2}, m_{1}, \ldots, m_{n-2}\right)= & \frac{(4 \pi)^{\epsilon}}{\Gamma(-\epsilon)} \int d \mu^{2}\left(\mu^{2}\right)^{-1-\epsilon} \int \frac{d^{4} \widetilde{\ell}}{(2 \pi)^{4}} \\
& \frac{1}{\left(\widetilde{\ell^{2}}-M_{1}^{2}-\mu^{2}\right)\left((\widetilde{\ell}-K)^{2}-M_{2}^{2}-\mu^{2}\right) \prod_{j=1}^{n-2}\left(\left(\widetilde{\ell}-P_{j}\right)^{2}-m_{j}^{2}-\mu^{2}\right)} .
\end{aligned}
$$

Following the setup of [19], we decompose the 4-momentum into a null component and a component proportional to the cut momentum $K$.

$$
\tilde{\ell}=\ell+z K, \quad \ell^{2}=0, \quad \Longrightarrow \int d^{4} \widetilde{\ell}=\int d z d^{4} \ell \delta^{+}\left(\ell^{2}\right)(2 \ell \cdot K)
$$

We define the "signature of the cut," $\Delta\left[K, M_{1}, M_{2}\right]$, as

$$
\Delta\left[K, M_{1}, M_{2}\right] \equiv\left(K^{2}\right)^{2}+\left(M_{1}^{2}\right)^{2}+\left(M_{2}^{2}\right)^{2}-2 K^{2} M_{1}^{2}-2 K^{2} M_{2}^{2}-2 M_{1}^{2} M_{2}^{2},
$$

and a dimensionless parameter $u$ to be

$$
u \equiv \frac{4 K^{2} \mu^{2}}{\Delta\left[K, M_{1}, M_{2}\right]}
$$

Then

$$
\int d \mu^{2}\left(\mu^{2}\right)^{-1-\epsilon} \rightarrow\left(\frac{\Delta\left[K, M_{1}, M_{2}\right]}{4 K^{2}}\right)^{-\epsilon} \int_{0}^{1} d u u^{-1-\epsilon}
$$

Since $\frac{(4 \pi)^{\epsilon}}{(2 \pi)^{4} \Gamma(-\epsilon)}\left(\frac{\Delta\left[K, M_{1}, M_{2}\right]}{4 K^{2}}\right)^{-\epsilon}$ is a universal factor on both sides (basis and amplitude) of the cut calculation, we neglect it henceforth.

Thus, we have rewritten the integral as

$$
\begin{aligned}
I_{n}\left(M_{1}, M_{2}, m_{1}, \ldots, m_{n-2}\right)= & \int_{0}^{1} d u u^{-1-\epsilon} \int d z d^{4} \ell \delta^{+}\left(\ell^{2}\right) \\
& (2 \ell \cdot K) \frac{1}{\left.\left(\widetilde{\ell^{2}}-M_{1}^{2}-\mu^{2}\right)\left((\widetilde{\ell}-K)^{2}-M_{2}^{2}-\mu^{2}\right) \prod_{j=1}^{n-2}\left(\widetilde{\ell}-P_{j}\right)^{2}-m_{j}^{2}-\mu^{2}\right)}(2.10)
\end{aligned}
$$

where $\mu^{2}$ is related to $u$ through (2.9). 


\subsection{Kinematics and the domain of integration}

In this subsection we determine the integration domain.

Assuming $K^{2} \neq 0$, we choose the frame where $\vec{K}=(K, 0,0,0), \widetilde{\ell}=(x, y, 0,0)$. Then the second cut propagator is $\widetilde{\ell}-\vec{K}=(x-K, y, 0,0)$. From the on-shell conditions,

$$
x^{2}-y^{2}=M_{1}^{2}+\mu^{2}, \quad(x-K)^{2}-y^{2}=M_{2}^{2}+\mu^{2},
$$

we solve for $x$ and $y$ to find

$$
x=\frac{K^{2}+M_{1}^{2}-M_{2}^{2}}{2 K}, \quad y= \pm \sqrt{x^{2}-M_{1}^{2}-\mu^{2}} .
$$

The requirement that $y$ has a real solution is the following constraint:

$$
\mu^{2} \leq \frac{\Delta\left[K, M_{1}, M_{2}\right]}{4 K^{2}}
$$

with the definition of $\Delta\left[K, M_{1}, M_{2}\right]$ given in (2.8). The condition (2.12) also requires the right hand side to be positive, which can be arranged by working in a region with sufficiently large cut momentum, $K>M_{1}+M_{2}$. Then the physical constraint (2.12) restricts $u$ to lie in a unit interval:

$$
u \in[0,1] .
$$

After using (2.7) to decompose the vector $\widetilde{\ell}=\ell+z K$, the lightlike condition $\ell^{2}=0$ becomes $(x-z K)^{2}=y^{2}$, or

$$
z^{2} K^{2}-z\left(K^{2}+M_{1}^{2}-M_{2}^{2}\right)+\left(M_{1}^{2}+\mu^{2}\right)=0
$$

Solving this equation, we find

$$
z=\frac{\left(K^{2}+M_{1}^{2}-M_{2}^{2}\right) \pm \sqrt{\Delta\left[K, M_{1}, M_{2}\right]-4 K^{2} \mu^{2}}}{2 K^{2}} .
$$

In the kinematic region of this cut, $K>0$. Then the positive-light-cone condition $\delta^{+}\left(\ell^{2}\right)$ is equivalent to $x-z K>0$. Consequently, exactly one of the two solutions (2.14) for $z$ is selected. Specifically,

$$
z=\frac{\left(K^{2}+M_{1}^{2}-M_{2}^{2}\right)-\sqrt{\Delta\left[K, M_{1}, M_{2}\right]-4 K^{2} \mu^{2}}}{2 K^{2}},
$$

which we rewrite as

$$
z=\frac{a-b \sqrt{1-u}}{2}
$$

where we have defined two useful parameters:

$$
a \equiv \frac{K^{2}+M_{1}^{2}-M_{2}^{2}}{K^{2}}, \quad b \equiv \frac{\sqrt{\Delta\left[K, M_{1}, M_{2}\right]}}{K^{2}} .
$$

In the massless limit $a=b=1$. 


\subsection{First steps: separating the four-dimensional integral}

Now we are ready to discuss the cut integral. Consider the two delta functions of the cut propagators,

$$
\delta\left(\widetilde{\ell}^{2}-M_{1}^{2}-\mu^{2}\right) \delta\left((\widetilde{\ell}-K)^{2}-M_{2}^{2}-\mu^{2}\right) .
$$

Inside the integral, we perform the following manipulations:

$$
\begin{aligned}
& \delta\left(\widetilde{\ell}^{2}-M_{1}^{2}-\mu^{2}\right) \delta\left((\widetilde{\ell}-K)^{2}-M_{2}^{2}-\mu^{2}\right) \\
& =\delta\left(\widetilde{\ell}^{2}-M_{1}^{2}-\mu^{2}\right) \delta\left(K^{2}-2 \widetilde{\ell} \cdot K+M_{1}^{2}-M_{2}^{2}\right) \\
& =\delta\left(z^{2} K^{2}+z(2 \ell \cdot K)-M_{1}^{2}-\mu^{2}\right) \delta\left((1-2 z) K^{2}-2 \ell \cdot K+M_{1}^{2}-M_{2}^{2}\right) \\
& =\delta\left(z(1-z) K^{2}+z\left(M_{1}^{2}-M_{2}^{2}\right)-M_{1}^{2}-\mu^{2}\right) \delta\left((1-2 z) K^{2}-2 \ell \cdot K+M_{1}^{2}-M_{2}^{2}\right)
\end{aligned}
$$

In the last step we used the second delta function to find $2 \ell \cdot K=(1-2 z) K^{2}+M_{1}^{2}-M_{2}^{2}$. We will make this substitution in the measure (2.7). At this point the first delta function is independent of $\ell$. The cut part of the integral now takes the form

$$
\begin{aligned}
& \int_{0}^{1} d u u^{-1-\epsilon} \int d z\left((1-2 z) K^{2}+M_{1}^{2}-M_{2}^{2}\right) \delta\left(z(1-z) K^{2}+z\left(M_{1}^{2}-M_{2}^{2}\right)-M_{1}^{2}-\mu^{2}\right) \\
& \int d^{4} \ell \delta^{+}\left(\ell^{2}\right) \delta\left((1-2 z) K^{2}-2 \ell \cdot K+M_{1}^{2}-M_{2}^{2}\right) .
\end{aligned}
$$

The second line is the 4-dimensional phase space integration, which can be performed in various ways, as discussed in [19]. We can integrate out $z$ using the delta-function in the first line. Here we need to account for the factor

$$
\frac{\partial}{\partial z}\left(z(1-z) K^{2}+z\left(M_{1}^{2}-M_{2}^{2}\right)-M_{1}^{2}-\mu^{2}\right)=(1-2 z) K^{2}+\left(M_{1}^{2}-M_{2}^{2}\right)
$$

which serves to cancel the first factor of the first line of (2.18). Finally, we arrive at the expression

$$
\int_{0}^{1} d u u^{-1-\epsilon} \int d^{4} \ell \delta^{+}\left(\ell^{2}\right) \delta\left((1-2 z) K^{2}-2 \ell \cdot K+M_{1}^{2}-M_{2}^{2}\right)
$$

where $z$ is related to $u$ by (2.16).

\section{Recursion and reduction formulas with mass}

In this section we compute the massive analogs of the recursion and reduction formulas for master integrals presented in [19] and derived in detail in [20]. It is not hard to check that the results of this section reproduce the massless results when we set all $m_{i}=0$ (and hence $a=b=1$ in (2.17)).

We refer to 20] rather than review all details of the setup here. However, we do need to remind the reader that the results for cuts of these basis integrals were derived with spinor integration, in which the massless 4-dimensional vector $\ell$ is rewritten as

$$
\ell=t \lambda \widetilde{\lambda}
$$


and the measure transforms as

$$
\int d^{4} \ell \delta^{(+)}\left(\ell^{2}\right)(\bullet)=\int_{0}^{\infty} d t t \int_{\tilde{\lambda}=\bar{\lambda}}\langle\lambda, d \lambda\rangle[\tilde{\lambda}, d \tilde{\lambda}](\bullet) .
$$

Here $t$ ranges over the positive real line, and $\lambda, \widetilde{\lambda}$ are homogeneous spinors, also written respectively as $|\ell\rangle, \mid \ell]$ in many expressions involving spinor products. The first step in spinor integration is to integrate over the variable $t$ simply by solving the delta function of the second cut propagator. In this section, we sketch the beginnings of certain derivations by writing the integrand before and after this $t$-integration-which, we emphasize, is not true integration.

Spinor integration proceeds by writing the integrand as a total derivative plus delta functions using "holomorphic anomaly" formulas, and finally identifying the contributions of delta functions as residues of a complex function. For an exposition of this technique, we refer the reader to [6, 7].

\subsection{Bubble}

The cut bubble is exactly the integral described in the previous section without any additional factors. We just need to do the four-dimensional integral.

$$
\begin{aligned}
& \int d z d^{4} \ell \delta^{+}\left(\ell^{2}\right)(2 \ell \cdot K) \delta\left(z(1-z) K^{2}+z\left(M_{1}^{2}-M_{2}^{2}\right)-M_{1}^{2}-\mu^{2}\right) \delta\left((1-2 z) K^{2}-2 \ell \cdot K+M_{1}^{2}-M_{2}^{2}\right) \\
= & \int d z d^{4} \ell \delta^{+}\left(\ell^{2}\right)\left((1-2 z) K^{2}+M_{1}^{2}-M_{2}^{2}\right) \times \\
& \delta\left(z(1-z) K^{2}+z\left(M_{1}^{2}-M_{2}^{2}\right)-M_{1}^{2}-\mu^{2}\right) \delta\left((1-2 z) K^{2}-2 \ell \cdot K+M_{1}^{2}-M_{2}^{2}\right) .
\end{aligned}
$$

After phase space integration we are left with

$$
\frac{(1-2 z) K^{2}+M_{1}^{2}-M_{2}^{2}}{K^{2}}=b \sqrt{1-u}
$$

where we have put in $z$ given by (2.16). So, the cut is

$$
C\left[I_{2}\left(M_{1}, M_{2} ; K\right)\right]=b \int_{0}^{1} d u u^{-1-\epsilon} \sqrt{1-u},
$$

where $b$ is given in (2.17). This factor of $b$ is where this expression differs from the massless case.

Recursion relation: Now we define a sequence of functions indexed by nonnegative integers $n$ :

$$
\operatorname{Bub}^{(n)} \equiv \int_{0}^{1} d u u^{-1-\epsilon} u^{n} \sqrt{1-u} .
$$

Note that the physical cut is related to the zeroth function by a factor of $b$ :

$$
C\left[I_{2}\left(M_{1}, M_{2} ; K\right)\right]=b \mathrm{Bub}^{(0)} .
$$

Because the definition (3.5) is identical to the massless case, we derive exactly the same recursion formula as in [19, 20]:

$$
\operatorname{Bub}^{(n)}=F_{2 \rightarrow 2}^{(n)} \mathrm{Bub}^{(0)}
$$


where the form factor is

$$
F_{2 \rightarrow 2}^{(n)}=\frac{(-\epsilon)_{\frac{3}{2}}}{(n-\epsilon)_{\frac{3}{2}}} .
$$

Here $(x)_{n}=\Gamma(x+n) / \Gamma(x)$ is the Pochhammer symbol.

Written in a form suitable for reading master integrands, we have the result

$$
b \int_{0}^{1} d u u^{-1-\epsilon} u^{n} \sqrt{1-u}=F_{2 \rightarrow 2}^{(n)} C\left[I_{2}\left(M_{1}, M_{2} ; K\right)\right] .
$$

\subsection{Triangle}

The integrand to start with is

$$
\frac{\delta\left(\widetilde{\ell^{2}}-M_{1}^{2}-\mu^{2}\right) \delta\left(\left(\widetilde{\ell}-K_{1}\right)^{2}-M_{2}^{2}-\mu^{2}\right)}{\left(\left(\widetilde{\ell}+K_{3}\right)^{2}-m_{1}^{2}-\mu^{2}\right)} .
$$

After $t$-integration we get

$$
-\left((1-2 z)+\frac{M_{1}^{2}-M_{2}^{2}}{K_{1}^{2}}\right) \frac{1}{\left\langle\ell\left|K_{1}\right| \ell\right]\langle\ell|Q| \ell]}=-b \sqrt{1-u} \frac{1}{\left\langle\ell\left|K_{1}\right| \ell\right]\langle\ell|Q| \ell]},
$$

where

$$
Q=\left((1-2 z)+\frac{M_{1}^{2}-M_{2}^{2}}{K_{1}^{2}}\right) K_{3}+\frac{K_{3}^{2}+M_{1}^{2}-m_{1}^{2}+z\left(2 K_{1} \cdot K_{3}\right)}{K_{1}^{2}} K_{1} .
$$

The four-dimensional integral gives

$$
-\frac{1}{\sqrt{\Delta_{3}}}\left((1-2 z)+\frac{M_{1}^{2}-M_{2}^{2}}{K_{1}^{2}}\right) \ln \left(\frac{-\left(2 K_{1} \cdot Q\right)+\sqrt{\Delta_{3}}}{-\left(2 K_{1} \cdot Q\right)-\sqrt{\Delta_{3}}}\right),
$$

where

$$
\Delta_{3}=4\left(\left(K_{1} \cdot Q\right)^{2}-K_{1}^{2} Q^{2}\right)
$$

The ingredients for (3.12) are

$$
\begin{aligned}
K_{1} \cdot Q & =\frac{K_{1}^{2}+M_{1}^{2}-M_{2}^{2}}{K_{1}^{2}}\left(K_{1} \cdot K_{3}\right)+\left(K_{3}^{2}+M_{1}^{2}-m_{1}^{2}\right) \\
Q^{2} & =\frac{\left(2 K_{1}^{2}\left(K_{3}^{2}+M_{1}^{2}-m_{1}^{2}\right)+\left(K_{1}^{2}+M_{1}^{2}-M_{2}^{2}\right)\left(2 K_{1} \cdot K_{3}\right)\right)^{2}-(1-u) \Delta_{3 ; m=0} \Delta\left[K_{1}, M_{1}, M_{2}\right]}{4\left(K_{1}^{2}\right)^{3}}
\end{aligned}
$$

where

$$
\Delta_{3 ; m=0} \equiv 4\left(\left(K_{1} \cdot K_{3}\right)^{2}-K_{1}^{2} K_{3}^{2}\right),
$$

which we recognize as the signature of a triangle with massless propagators. 
We can now see that

$$
\Delta_{3}=\frac{(1-u) \Delta_{3 ; m=0} \Delta\left[K_{1}, M_{1}, M_{2}\right]}{\left(K_{1}^{2}\right)^{2}}=b^{2}(1-u) \Delta_{3 ; m=0} .
$$

It is interesting to see that $\Delta_{3}$ is built from the factors $\Delta\left[K_{1}, M_{1}, M_{2}\right]$, the signature of the cut, and $\Delta_{3 ; m=0}$, the signature of the triangle.

Now we define

$$
Z \equiv-\frac{\left(2 K_{1} \cdot Q\right) K_{1}^{2}}{\sqrt{\Delta_{3 ; m=0} \Delta\left[K_{1}, M_{1}, M_{2}\right]}}
$$

Then we have

$$
\begin{aligned}
C\left[I_{3}\left(M_{1}, M_{2}, m_{1} ; K_{1}, K_{3}\right)\right] & =\int_{0}^{1} d u u^{-1-\epsilon}(-b \sqrt{1-u}) \frac{1}{\sqrt{\Delta_{3}}} \ln \left(\frac{Z+\sqrt{1-u}}{Z-\sqrt{1-u}}\right) \\
& =\int_{0}^{1} d u u^{-1-\epsilon}\left(-\frac{1}{\sqrt{\Delta_{3 ; m=0}}}\right) \ln \left(\frac{Z+\sqrt{1-u}}{Z-\sqrt{1-u}}\right) .
\end{aligned}
$$

This is the same expression as in the massless case, but now with a different $Z$.

Recursion/reduction relation: We define the integrals

$$
\operatorname{Tri}^{(n)}(Z) \equiv \int_{0}^{1} d u u^{-1-\epsilon} u^{n} \ln \left(\frac{Z+\sqrt{1-u}}{Z-\sqrt{1-u}}\right),
$$

where the parameter $Z$ is defined as in $(3.15)$. The physical cut integral is

$$
C\left[I_{3}\left(M_{1}, M_{2}, m_{1} ; K_{1}, K_{3}\right)\right]=-\frac{1}{\sqrt{\Delta_{3 ; m=0}}} \operatorname{Tri}^{(0)}(Z) .
$$

In this case, our cut triangle functions (3.17) do depend on the cut-propagator masses $M_{1}$ and $M_{2}$ via $Z$. Apart from the generalized definition of $Z$, the formula is the same as in the massless case. Therefore, we derive the same recursion and reduction formulas as in [20], namely

$$
\operatorname{Tri}^{(n)}(Z)=F_{3 \rightarrow 3}^{(n)}(Z) \operatorname{Tri}^{(0)}(Z)+\widetilde{F}_{3 \rightarrow 2}^{(n)}(Z) \operatorname{Bub}^{(0)},
$$

where the two form factors are given by

$$
\begin{aligned}
& F_{3 \rightarrow 3}^{(n)}(Z)=\frac{-\epsilon}{n-\epsilon}\left(1-Z^{2}\right)^{n} \\
& \widetilde{F}_{3 \rightarrow 2}^{(n)}(Z)=\frac{(-\epsilon)_{\frac{3}{2}}}{n-\epsilon} \sum_{k=1}^{n} \frac{2 Z\left(1-Z^{2}\right)^{n-k}}{(k-\epsilon)_{\frac{1}{2}}} .
\end{aligned}
$$

These are functions of the variable $Z$, defined for a given triangle by (3.15). 
Equation (3.19) is not yet in the most applicable form. We return to the language of physical cuts by including the factor $-1 / \sqrt{\Delta_{3, m=0}}$ from (3.18). The recursion/reduction formula that we need is thus:

$$
\begin{aligned}
& \int_{0}^{1} d u u^{-1-\epsilon} u^{n}\left[-\frac{1}{\sqrt{\Delta_{3 ; m=0}}} \ln \left(\frac{Z+\sqrt{1-u}}{Z-\sqrt{1-u}}\right)\right]= \\
& F_{3 \rightarrow 3}^{(n)}(Z) C\left[I_{3}\left(M_{1}, M_{2}, m_{1} ; K_{1}, K_{3}\right)\right]+F_{3 \rightarrow 2}^{(n)}\left(K_{1}, K_{3}\right) C\left[I_{2}\left(M_{1}, M_{2} ; K_{1}\right)\right],
\end{aligned}
$$

where

$$
\left.F_{3 \rightarrow 2}^{(n)}\left(K_{1}, K_{3}\right)\right)=-\frac{1}{b \sqrt{\Delta_{3 ; m=0}}} \widetilde{F}_{3 \rightarrow 2}^{(n)}(Z)
$$

\subsection{Box}

The integrand to start with is

$$
\frac{\delta\left(\widetilde{\ell^{2}}-M_{1}^{2}-\mu^{2}\right) \delta\left((\widetilde{\ell}-K)^{2}-M_{2}^{2}-\mu^{2}\right)}{\left(\left(\widetilde{\ell}-P_{1}\right)^{2}-m_{1}^{2}-\mu^{2}\right)\left(\left(\widetilde{\ell}-P_{2}\right)^{2}-m_{2}^{2}-\mu^{2}\right)} .
$$

We define another useful mass-dependent parameter:

$$
a_{i} \equiv \frac{P_{i}^{2}+M_{1}^{2}-m_{i}^{2}}{K^{2}}
$$

After $t$-integration we get

$$
\frac{b \sqrt{1-u}}{K^{2}} \frac{1}{\left\langle\ell\left|Q_{1}\right| \ell\right]\left\langle\ell\left|Q_{2}\right| \ell\right]},
$$

where

$$
Q_{i}=(-b \sqrt{1-u}) P_{i}+\left(a_{i}-\frac{P_{i} \cdot K}{K^{2}}(a-b \sqrt{1-u})\right) K
$$

Now the procedure is the same as in the massless case, but with this more general definition of $Q_{i}$.

Define

$$
\begin{aligned}
R_{i} & =\left.Q_{i}\right|_{u=0} \\
& =-b P_{i}+\left(a_{i}-\frac{P_{i} \cdot K}{K^{2}}(a-b)\right) K
\end{aligned}
$$

The physical cut is

$$
\begin{aligned}
& C\left[I_{4}\left(M_{1}, M_{2}, m_{1}, m_{2} ; K, P_{1}, P_{2}\right)=\right. \\
& \int_{0}^{1} d u u^{-1-\epsilon} \frac{b}{2 K^{2}} \frac{1}{\sqrt{B-A u}} \ln \left(\frac{D-C u+\sqrt{1-u} \sqrt{B-A u}}{D-C u-\sqrt{1-u} \sqrt{B-A u}}\right),
\end{aligned}
$$


where

$$
\begin{array}{cll}
A=-\frac{b^{4}}{K^{2}} \operatorname{det}\left(\begin{array}{ccc}
P_{1}^{2} & P_{1} \cdot P_{2} & P_{1} \cdot K \\
P_{1} \cdot P_{2} & P_{2}^{2} & P_{2} \cdot K \\
P_{1} \cdot K & P_{2} \cdot K & K^{2}
\end{array}\right), & C=\frac{b^{2}}{K^{2}} \operatorname{det}\left(\begin{array}{cc}
P_{1} \cdot P_{2} & P_{1} \cdot K \\
P_{2} \cdot K & K^{2}
\end{array}\right), \\
B=-\operatorname{det}\left(\begin{array}{cc}
R_{1}^{2} & R_{1} \cdot R_{2} \\
R_{1} \cdot R_{2} & R_{2}^{2}
\end{array}\right), & D=R_{1} \cdot R_{2} .
\end{array}
$$

Here again, the form of (3.28) differs from the one in massless case only by the factor of $b$.

Recursion/reduction relation: We define

$$
\operatorname{Box}^{(n)}(A, B, C, D) \equiv \int_{0}^{1} d u u^{-1-\epsilon} \frac{u^{n}}{\sqrt{B-A u}} \ln \left(\frac{D-C u+\sqrt{1-u} \sqrt{B-A u}}{D-C u-\sqrt{1-u} \sqrt{B-A u}}\right) .
$$

The physical cut is related to the zeroth function in (3.30) by

$$
C\left[I_{4}\left(M_{1}, M_{2}, m_{1}, m_{2} ; K, P_{1}, P_{2}\right)\right]=\frac{b}{2 K^{2}} \operatorname{Box}^{(0)}(A, B, C, D)
$$

if $A, B, C, D$ are defined as in (3.29).

In Table (3.31) we have listed which kinds of triangles a box with given cut would reduce to (the propagator mass must also be correctly identified):

\begin{tabular}{|c|c|c|c|c|}
\hline Box Cut $K$ & $P_{1}$ & $P_{2}$ & Triangle One's $\left(K_{1}, K_{3}\right)$ & Triangle Two's $\left(K_{1}, K_{3}\right)$ \\
\hline$K_{1}$ & $K_{12}$ & $-K_{4}$ & $\left(K_{1}, K_{34}\right)$ & $\left(K_{1}, K_{4}\right)$ \\
\hline$K_{2}$ & $K_{23}$ & $-K_{1}$ & $\left(K_{2}, K_{41}\right)$ & $\left(K_{2}, K_{1}\right)$ \\
\hline$K_{3}$ & $K_{34}$ & $-K_{2}$ & $\left(K_{3}, K_{12}\right)$ & $\left(K_{3}, K_{2}\right)$ \\
\hline$K_{4}$ & $K_{41}$ & $-K_{3}$ & $\left(K_{4}, K_{23}\right)$ & $\left(K_{4}, K_{3}\right)$ \\
\hline$K_{12}$ & $K_{1}$ & $-K_{4}$ & $\left(K_{34}, K_{2}\right)$ & $\left(K_{12}, K_{4}\right)$ \\
\hline$K_{23}$ & $K_{2}$ & $-K_{1}$ & $\left(K_{41}, K_{3}\right)$ & $\left(K_{23}, K_{1}\right)$ \\
\hline
\end{tabular}

Boxes are related to triangles and bubbles in the reduction formulas. In these relations we make use of a quantity combining variables of the box and the associated triangles to Table (3.31):

$$
\begin{gathered}
C_{Z_{i}} \equiv\left(Z_{i}^{2}-1\right) C+D \\
\operatorname{Box}^{(n)}(A, B, C, D)=F_{4 \rightarrow 4}^{(n)}(A, B) \operatorname{Box}^{(0)}(A, B, C, D)+\widetilde{F}_{4 \rightarrow 3}^{(n)}\left(A, B, C, D ; Z_{1}\right) \operatorname{Tri}^{(0)}\left(Z_{1}\right) \\
+\widetilde{F}_{4 \rightarrow 3}^{(n)}\left(A, B, C, D ; Z_{2}\right) \operatorname{Tri}^{(0)}\left(Z_{2}\right)+\widetilde{F}_{4 \rightarrow 2}^{(n)}\left(A, B, C, D ; Z_{i}\right) \mathrm{Bub}^{(0)},
\end{gathered}
$$

where the form factors are given by

$$
F_{4 \rightarrow 4}^{(n)}(A, B)=\frac{(-\epsilon)_{\frac{1}{2}}}{(n-\epsilon)_{\frac{1}{2}}}\left(\frac{B}{A}\right)^{n},
$$




$$
\begin{aligned}
& \widetilde{F}_{4 \rightarrow 3}^{(n)}\left(A, B, C, D ; Z_{i}\right)=-\frac{C_{Z_{i}}}{(n-\epsilon)_{\frac{1}{2}} A Z_{i}} \sum_{k=1}^{n}(k-1-\epsilon)_{\frac{1}{2}}\left(\frac{B}{A}\right)^{n-k} F_{3 \rightarrow 3}^{(k-1)}\left(Z_{i}\right), \\
& \widetilde{F}_{4 \rightarrow 2}^{(n)}\left(A, B, C, D ; Z_{i}\right)=-\frac{\Gamma(n-\epsilon)}{\Gamma(n+1 / 2-\epsilon)} \frac{1}{A} \\
& \times \sum_{k=1}^{n}(k-1-\epsilon)_{\frac{1}{2}}\left(\frac{B}{A}\right)^{n-k}\left(\frac{C_{Z_{1}}}{Z_{1}} \widetilde{F}_{3 \rightarrow 2}^{(k-1)}\left(Z_{1}\right)+\frac{C_{Z_{2}}}{Z_{2}} \widetilde{F}_{3 \rightarrow 2}^{(k-1)}\left(Z_{2}\right)\right) .
\end{aligned}
$$

Again (3.33) is not the final formula we are after. To get the proper physical result for identifying integrands, we need to replace the kinematic factor $b / 2 K^{2}$. The result is

$$
\begin{aligned}
\int_{0}^{1} d u & u^{-1-\epsilon} u^{n}\left[\frac{b}{2 K^{2} \sqrt{B-A u}} \ln \left(\frac{D-C u+\sqrt{1-u} \sqrt{B-A u}}{D-C u-\sqrt{1-u} \sqrt{B-A u}}\right)\right]= \\
& F_{4 \rightarrow 4}^{(n)}(A, B) C\left[I_{4}\left(M_{1}, M_{2}, m_{1}, m_{2} ; K, P_{1}, P_{2}\right)\right] \\
& +\sum_{i=1}^{2} F_{4 \rightarrow 3}^{(n)}\left(A, B, C, D ; Z_{i}\right) C\left[I_{3}\left(M_{1}, M_{2}, m_{1}^{(i)} ; K_{1}^{(i)}, K_{3}^{(i)}\right)\right] \\
& +F_{4 \rightarrow 2}^{(n)}\left(A, B, C, D ; Z_{i}\right) C\left[I_{2}\left(M_{1}, M_{2} ; K\right)\right],
\end{aligned}
$$

where for the triangles, $K_{1}^{(i)}$ and $K_{3}^{(i)}$ are given by Table (3.31), the mass $m_{1}^{(1)}$ must also be interpreted correctly according to the same table, and the form factors are

$$
\begin{aligned}
& F_{4 \rightarrow 3}^{(n)}\left(A, B, C, D ; Z_{i}\right)=-\frac{b \sqrt{\Delta_{3}^{(i)}}}{2 K^{2}} \widetilde{F}_{4 \rightarrow 3}^{(n)}\left(A, B, C, D ; Z_{i}\right), \\
& F_{4 \rightarrow 2}^{(n)}\left(A, B, C, D ; Z_{i}\right)=\frac{1}{2 K^{2}} \widetilde{F}_{4 \rightarrow 2}^{(n)}\left(A, B, C, D ; Z_{i}\right) .
\end{aligned}
$$

\subsection{Pentagon}

The integrand is

$$
\frac{\delta\left(\widetilde{\ell^{2}}-M_{1}^{2}-\mu^{2}\right) \delta\left((\widetilde{\ell}-K)^{2}-M_{2}^{2}-\mu^{2}\right)}{\left(\left(\widetilde{\ell}-P_{1}\right)^{2}-m_{1}^{2}-\mu^{2}\right)\left(\left(\widetilde{\ell}-P_{2}\right)^{2}-m_{2}^{2}-\mu^{2}\right)\left(\left(\widetilde{\ell}-P_{3}\right)^{2}-m_{3}^{2}-\mu^{2}\right)} .
$$

The physical cut is

$$
C\left[I_{5}\left(M_{1}, M_{2}, m_{1}, m_{2}, m_{3} ; K, P_{1}, P_{2}, P_{3}\right)\right]=\int_{0}^{1} d u u^{-1-\epsilon} \int\langle\ell d \ell\rangle[\ell d \ell] \frac{b \sqrt{1-u}\langle\ell|K| \ell]}{\left(K^{2}\right)^{2}\left\langle\ell\left|Q_{1}\right| \ell\right]\left\langle\ell\left|Q_{2}\right| \ell\right]\left\langle\ell\left|Q_{3}\right| \ell\right]},
$$

where

$$
Q_{i}=-(b \sqrt{1-u}) P_{i}+\frac{P_{i}^{2}+M_{1}^{2}-m_{i}^{2}-2 z\left(K \cdot P_{i}\right)}{K^{2}} K .
$$

The total integral (apart from the universal prefactor) is

$$
b \int_{0}^{1} d u u^{-1-\epsilon} \sqrt{1-u} \int\langle\ell d \ell\rangle[\ell d \ell] \frac{\langle\ell|K| \ell]}{\left(K^{2}\right)^{2}\left\langle\ell\left|Q_{1}\right| \ell\right]\left\langle\ell\left|Q_{2}\right| \ell\right]\left\langle\ell\left|Q_{3}\right| \ell\right]} .
$$


Apart from the factor of $b$ and the modified definition of $Q_{i}$, the analysis proceeds as in the massless case [20]. Thus we can cite the result directly as

$$
\begin{aligned}
& C\left[I_{5}\left(M_{1}, M_{2}, m_{1}, m_{2}, m_{3} ; K, P_{1}, P_{2}, P_{3}\right)\right]=-b \int_{0}^{1} d u u^{-1-\epsilon} \frac{\sqrt{1-u}}{\left(K^{2}\right)^{2}} \\
& \left(\frac{S\left[Q_{3}, Q_{2}, Q_{1}, K\right]}{4 \sqrt{\left(Q_{3} \cdot Q_{2}\right)^{2}-Q_{3}^{2} Q_{2}^{2}}} \ln \frac{Q_{3} \cdot Q_{2}-\sqrt{\left(Q_{3} \cdot Q_{2}\right)^{2}-Q_{3}^{2} Q_{2}^{2}}}{Q_{3} \cdot Q_{2}+\sqrt{\left(Q_{3} \cdot Q_{2}\right)^{2}-Q_{3}^{2} Q_{2}^{2}}}\right. \\
& +\frac{S\left[Q_{3}, Q_{1}, Q_{2}, K\right]}{4 \sqrt{\left(Q_{3} \cdot Q_{1}\right)^{2}-Q_{3}^{2} Q_{1}^{2}}} \ln \frac{Q_{3} \cdot Q_{1}-\sqrt{\left(Q_{3} \cdot Q_{1}\right)^{2}-Q_{3}^{2} Q_{1}^{2}}}{Q_{3} \cdot Q_{1}+\sqrt{\left(Q_{3} \cdot Q_{1}\right)^{2}-Q_{3}^{2} Q_{1}^{2}}} \\
& \left.+\frac{S\left[Q_{2}, Q_{1}, Q_{3}, K\right]}{4 \sqrt{\left(Q_{2} \cdot Q_{1}\right)^{2}-Q_{2}^{2} Q_{1}^{2}}} \ln \frac{Q_{2} \cdot Q_{1}-\sqrt{\left(Q_{2} \cdot Q_{1}\right)^{2}-Q_{2}^{2} Q_{1}^{2}}}{Q_{2} \cdot Q_{1}+\sqrt{\left(Q_{2} \cdot Q_{1}\right)^{2}-Q_{2}^{2} Q_{1}^{2}}}\right),
\end{aligned}
$$

where $S\left[Q_{3}, Q_{2}, Q_{1}, K\right]$ is a rational function defined as follows:

$$
S\left[Q_{2}, Q_{1}, Q_{3}, K\right]=\frac{T_{1}}{T_{2}}
$$

with

$$
T_{1}=-8 \operatorname{det}\left(\begin{array}{ccr}
Q_{3} \cdot K & Q_{2} \cdot K & Q_{1} \cdot K \\
Q_{2} \cdot Q_{3} & Q_{2}^{2} & Q_{2} \cdot Q_{1} \\
Q_{1} \cdot Q_{3} & Q_{2} \cdot Q_{1} & Q_{1}^{2}
\end{array}\right), \quad T_{2}=-4 \operatorname{det}\left(\begin{array}{lrr}
Q_{3}^{2} & Q_{2} \cdot Q_{3} & Q_{1} \cdot Q_{3} \\
Q_{2} \cdot Q_{3} & Q_{2}^{2} & Q_{2} \cdot Q_{1} \\
Q_{1} \cdot Q_{3} & Q_{2} \cdot Q_{1} & Q_{1}^{2}
\end{array}\right)
$$

Let us make a few comments on the behavior of pentagon cuts. There are three terms. Each term looks like a box signature multiplied by the factor $S[\bullet] /\left(2 K^{2}\right)$. It is significant that each function $S[\bullet]$ has the same denominator $T_{2}[\bullet]$, which does not depend on the order of the first three arguments. This can be considered as another signature of a cut-pentagon integral. This feature makes the reduction simple. Where we see a factor of $u^{n}$, we just need to write $u^{n} S=P(u)+A \sum_{i=1}^{3} S_{i}[\bullet]$ where $P(u)$ is a polynomial in $u$ and $A$ is constant in $u .^{2}$ The $A$ term will be the pentagon coefficient, while $P(u)$ indicates reduction to boxes. In the massless case, pentagons contribute to terms of $\mathcal{O}(\epsilon)$, so they can be neglected. However, in cases where propagators are massive or we wish to compute to higher orders in $\epsilon$, their contribution must be included.

\section{Formulas for coefficients from double cuts}

Systematic extraction of coefficients of master integrals from four-dimensional spinor integration techniques has been described in [6, 7], building on earlier techniques reviewed in [22, 23]. When applied to a specific amplitude in practice, there are choices to make regarding how to "split" the integrand in partial fractions as well as choosing arbitrary spinors on which the final answer does not depend.

\footnotetext{
${ }^{2}$ It is essential that because all three $S_{i}[\bullet]$ have same denominator $T_{2}$, after reduction we have the same $A$ multiplying all three of the $S_{i}[\bullet]$.
} 
In this section we present canonical choices to further simplify the method, aiming to automatize the heart of this procedure to allow for easy implementation into a computer program.

For brevity, we do not review the entire spinor integration technique here; instead we refer the reader to the explanations given in [6, 7]. The steps that concern us here come after the coordinate change and $t$-integration mentioned at the beginning of Section 3. At this point we have an integrand whose terms are rational functions of spinor products and homogeneous in the spinor integration variables. The following steps are to split the integrand into partial fractions (using Schouten identities), followed by identification of master integrals and integration over a single Feynman parameter. Finally we apply the holomorphic anomaly to complete the spinor integration by extracting residues.

Here we are able to give explicit algebraic functions for coefficients. In the appendix we give a more detailed discussion of how to evaluate these functions in practice.

A summary of the results this section may be found in Section 4.4.

\subsection{Canonical splitting}

Recall that our starting point is the product of the two (on-shell) tree-level amplitudes from each side of the unitarity cut. In spinor notation, this integrand takes the general form ${ }^{3}$

$$
\sum \frac{C \prod_{i=1}^{n_{1}}\left\langle\widetilde{a}_{i}|\ell| \widetilde{b}_{j}\right]}{\prod_{j=1}^{n_{2}}\left(\left(\widetilde{\ell}-P_{j}\right)^{2}-\mu^{2}\right)}
$$

Here $C$ is some expression that does not depend on any integration variable, and we have used the relation (2.7), $\tilde{\ell}=\ell+z K$, to rewrite the numerator. As we emphasized in the beginning of Section 3 , the $t$ integration is trivial because of the second delta function, $\delta\left((1-2 z) K^{2}-2 \ell \cdot K\right)$. Thus we can write the result of this step immediately:

$$
\frac{(1-2 z) K^{2}}{\langle\ell|K| \ell]^{2}} \sum\left(-\frac{(1-2 z) K^{2}}{\langle\ell|K| \ell]}\right)^{n_{1}} \frac{\langle\ell|K| \ell]^{n_{2}} C \prod_{i=1}^{n_{1}}\left\langle\widetilde{a}_{i} \ell\right\rangle\left[\ell \widetilde{b}_{i}\right]}{\prod_{j=1}^{n_{2}}\left\langle\ell\left|Q_{j}\right| \ell\right]} .
$$

So we see that, after integrating over $t$, the integrand for phase space integration is a sum of terms of the following form:

$$
I_{\text {term }}=\frac{G(\lambda) \prod_{j=1}^{n+k-2}\left[a_{j} \ell\right]}{\langle\ell|K| \ell]^{n} \prod_{i=1}^{k}\left\langle\ell\left|Q_{i}\right| \ell\right]}
$$

Here $G(\lambda)$ is some monomial in the holomorphic spinor only; hence it factorizes as $\prod\left\langle\ell c_{i}\right\rangle$ times a constant in $\ell$. The functions $a_{j}$ may depend on $\lambda$ as well, for example as $\left[a_{j} \mid=\left\langle\lambda|Q|\right.\right.$. But $a_{j}$ is the quantity we will need in order to split the term further and extract the residues from multiple poles.

We aim to describe all coefficients in terms of the quantities $K, Q_{i}, a_{j}$ and $G(\lambda)$.

\footnotetext{
${ }^{3}$ In this section we deal specifically with the case of massless propgators. The generalization to the massive case is straightforward, as in the previous sections.
} 
The term (4.1) depends as well on $n$ and $k$. The exponent $n$ is related to the type of master integral involved. Terms with $n=0$ contribute only to boxes and pentagons. Terms with $n=1$ contribute to triangles in addition, and terms with $n \geq 2$ contribute to all of these plus bubbles. If $n=0$ we can multiply both numerator and denominator by $\langle\ell|K| \ell]$, so we shall always assume $n \geq 1$.

The first step in our program is to isolate poles by splitting $I_{\text {term }}$ using the following partial fraction spinor identity:

$$
\frac{[a \ell]}{\left\langle\ell\left|Q_{1}\right| \ell\right]\left\langle\ell\left|Q_{2}\right| \ell\right]}=\frac{\left[a\left|Q_{1}\right| \ell\right\rangle}{\left\langle\ell\left|Q_{2} Q_{1}\right| \ell\right\rangle\left\langle\ell\left|Q_{1}\right| \ell\right]}+\frac{\left[a\left|Q_{2}\right| \ell\right\rangle}{\left\langle\ell\left|Q_{1} Q_{2}\right| \ell\right\rangle\left\langle\ell\left|Q_{2}\right| \ell\right]} .
$$

Because there are different factors in the denominator, there are different sequences of splitting leading to different but equivalent expressions. Here we choose a canonical sequence:

(1) First we keep $\langle\ell|K| \ell]^{n}$ untouched and split the factors $\left\langle\ell\left|Q_{i}\right| \ell\right]$ among themselves. At the end of this step each term takes the form $\frac{\widetilde{G}(\lambda)}{\langle\ell|K| \ell]^{n}\left\langle\ell\left|Q_{i}\right| \ell\right]}$.

(2) Then we split $\langle\ell|K| \ell]$ from $\left\langle\ell\left|Q_{i}\right| \ell\right]$ as often as necessary to get two types of terms, $\frac{\widetilde{G}_{m}(\lambda)}{\langle\ell|K| \ell]^{m}}$ and $\widetilde{F}_{i}(\lambda)$ $\frac{\tilde{F}_{i}(\lambda)}{\langle\ell|K| \ell|\left\langle\ell\left|Q_{i}\right| \ell\right]}$.

(3) Finally we will be left with

$$
\frac{G(\lambda) \prod_{j=1}^{n+k-2}\left[a_{j} \ell\right]}{\langle\ell|K| \ell]^{n} \prod_{i=1}^{k}\left\langle\ell\left|Q_{i}\right| \ell\right]}=\sum_{k=2}^{n} G_{k}(\lambda) \frac{\prod_{j=1}^{k-2}\left[b_{j} \ell\right]}{\langle\ell|K| \ell]^{k}}+\sum_{i=1}^{k} F_{i}(\lambda) \frac{1}{\langle\ell|K| \ell]\left\langle\ell\left|Q_{i}\right| \ell\right]} .
$$

Our task is to find expressions for $G_{k}(\lambda), F_{i}(\lambda)$ and $b_{j}$ in the most compact and simple form. The result is

$$
\begin{aligned}
F_{i}(\lambda) & =\left(\frac{G(\lambda) \prod_{s=1}^{n+k-2}\left[a_{s}\left|Q_{i}\right| \ell\right\rangle}{\left\langle\ell\left|K Q_{i}\right| \ell\right\rangle^{n-1} \prod_{t=1, t \neq i}^{k}\left\langle\ell\left|Q_{t} Q_{i}\right| \ell\right\rangle}\right), \\
G_{p}(\lambda) & =\sum_{i=1}^{k} \frac{G(\lambda) \prod_{s=1}^{k-1}\left[a_{s}\left|Q_{i}\right| \ell\right\rangle}{\prod_{t=1, t \neq i}^{k}\left\langle\ell\left|Q_{t} Q_{i}\right| \ell\right\rangle} \frac{\prod_{l=k}^{n-k-p}\left[a_{l}|K| \ell\right\rangle}{\left\langle\ell\left|Q_{i} K\right| \ell\right\rangle^{n+1-p}}, \quad p=2, \ldots, n \\
b_{j} & =a_{j+n+k-p} .
\end{aligned}
$$

While $F_{i}(\lambda)$ finds its simplest and most compact form in (4.4), $G_{p}(\lambda)$ in (4.5) may not be the simplest. For example, it can be shown that all terms with $1 /\langle\ell|K| \ell]^{n}$ can be summed into a single term. In general there are several terms with $1 /\langle\ell|K| \ell]^{a}$ with $2 \leq a<n$. As we discuss in Section 5.3, if we know that $n \leq 4$, we can work them out explicitly. However, in Section 4.3 we shall use a slightly different method to deal with these rational terms.

The identities necessary for carrying out steps (1) and (2) are the following, which may be proved by induction, making use of the basic splitting identity (4.2).

$$
\frac{\prod_{j=1}^{k-1}\left[a_{j} \ell\right]}{\prod_{i=1}^{k}\left\langle\ell\left|Q_{i}\right| \ell\right]}=\sum_{i=1}^{k} \frac{1}{\left\langle\ell\left|Q_{i}\right| \ell\right]} \frac{\prod_{j=1}^{k-1}\left[a_{j}\left|Q_{i}\right| \ell\right\rangle}{\prod_{m=1, m \neq i}^{k}\left\langle\ell\left|Q_{m} Q_{i}\right| \ell\right\rangle}
$$


$\frac{\prod_{j=1}^{n-1}\left[a_{j} \ell\right]}{\langle\ell|K| \ell]^{n}\langle\ell|Q| \ell]}=\frac{\prod_{j=1}^{n-1}\left[a_{j}|Q| \ell\right\rangle}{\langle\ell|K Q| \ell\rangle^{n-1}} \frac{1}{\langle\ell|K| \ell]\langle\ell|Q| \ell]}+\sum_{p=0}^{n-2}(-)^{n-p} \frac{\prod_{j=1}^{n-p-2}\left[a_{j}|Q| \ell\right\rangle\left[a_{n-p-1}|K| \ell\right\rangle \prod_{t=n-p}^{n-1}\left[a_{t} \ell\right]}{\langle\ell|K| \ell]^{p+2}\langle\ell|Q K| \ell\rangle^{n-p-1}}(4$

One necessary condition for the form (4.7) is that all the $Q_{i}$ are different, which is satisfied for generic momenta.

\subsection{Box and triangle coefficients}

In this subsection we derive a closed expression for box coefficients and systematize the derivation of triangle coefficients.

As we know from Section 3.3, box integrals and their coefficients may be labeled by momenta $P_{i}, P_{j}$ along with the cut momentum $K$. From these momenta we have defined $Q_{i}, Q_{j}$ as in (3.25).

The terms we need to consider are of the following form:

$$
\begin{aligned}
I^{(i)} & =\int F_{i}(\lambda) \frac{1}{\langle\ell|K| \ell]\left\langle\ell\left|Q_{i}\right| \ell\right]} \\
& =\int_{0}^{1} d x \int\langle\ell d \ell\rangle\left[d \ell \partial_{\ell}\right]\left(\frac{F_{i}(\lambda)[\eta \ell]}{\langle\ell|R| \ell]\langle\ell|R| \eta]}\right), \quad R=x Q_{i}+(1-x) K
\end{aligned}
$$

As discussed in the previous subsection, there will be two types of poles: those from $\langle\ell|K Q| \ell\rangle$ contribute to triangles, and those from $\left\langle\ell\left|Q_{j} Q_{i}\right| \ell\right\rangle$ contribute to boxes. Let us see precisely how these arise.

We can construct two massless momenta from $Q_{i}$ and $K$ :

$$
P_{1,2}^{(i)}=Q_{i}+x_{1,2}^{(i)} K
$$

where

$$
x_{1,2}^{(i)}=\frac{-2 Q_{i} \cdot K \pm \sqrt{\Delta^{(i)}}}{2 K^{2}}, \quad \Delta^{(i)}=\left(2 Q_{i} \cdot K\right)^{2}-4 Q_{i}^{2} K^{2}
$$

We make the choice $\eta=P_{1}^{(i)}$ and find that

$$
I^{(i)}=\int_{0}^{1} d x \int\langle\ell d \ell\rangle\left[d \ell \partial_{\ell}\right]\left(\frac{F_{i}(\lambda)\left[P_{1}^{(i)} \ell\right]}{\left\langle\ell P_{2}^{(i)}\right\rangle\left[P_{2}^{(i)} P_{1}^{(i)}\right]} \frac{\left(x_{1}^{(i)}-x_{2}^{(i)}\right)}{\langle\ell|R| \ell]\left(x\left(x_{1}^{(i)}+1\right)-1\right)}\right) .
$$

The poles in this expression come from $F_{i}(\lambda)$ and $\left\langle\ell P_{2}^{(i)}\right\rangle$. Note as well that since we have chosen $\eta=P_{1}^{(i)}$, the factor $\left[P_{1}^{(i)} \ell\right]$ appears in the numerator and therefore there is no contribution from the pole at $\left\langle\ell P_{1}^{(i)}\right\rangle$, which one might naively expect from a factor of $\left\langle\ell\left|K Q_{i}\right| \ell\right\rangle$ in the denominator of $F_{i}(\lambda)$.

Apply a partial fraction expansion to the $x$-dependent factors in the denominator of the integral. The result is

$$
I^{(i)}=\int_{0}^{1} d x \int\langle\ell d \ell\rangle\left[d \ell \partial_{\ell}\right]\left(\frac{F_{i}(\lambda)}{\left\langle\ell\left|Q_{i} K\right| \ell\right\rangle}\left(-\frac{\left(x_{1}^{(i)}+1\right)}{x\left(x_{1}^{(i)}+1\right)-1}+\frac{\left\langle\ell\left|Q_{i}-K\right| \ell\right]}{x\left\langle\ell\left|Q_{i}-K\right| \ell\right]+\langle\ell|K| \ell]}\right)\right),
$$


where we have used the fact that

$$
\left\langle\ell\left|Q_{i} K\right| \ell\right\rangle=\frac{\left\langle\ell P_{1}^{(i)}\right\rangle\left[P_{1}^{(i)} P_{2}^{(i)}\right]\left\langle\ell P_{2}^{(i)}\right\rangle}{\left(x_{1}^{(i)}-x_{2}^{(i)}\right)} .
$$

First term of (4.12): Let us consider the two terms of (4.12) separately. The first is equal to

$$
\begin{aligned}
I_{1}^{(i)} & \equiv \int_{0}^{1} d x \int\langle\ell d \ell\rangle\left[d \ell \partial_{\ell}\right]\left(\frac{F_{i}(\lambda)}{\left\langle\ell\left|Q_{i} K\right| \ell\right\rangle}\left(-\frac{\left(x_{1}^{(i)}+1\right)}{x\left(x_{1}^{(i)}+1\right)-1}\right)\right) \\
& =\int\langle\ell d \ell\rangle\left[d \ell \partial_{\ell}\right]\left(\frac{F_{i}(\lambda)}{\left\langle\ell\left|Q_{i} K\right| \ell\right\rangle}\left(-\ln \left(-x_{1}^{(i)}\right)\right)\right) \\
& =\int\langle\ell d \ell\rangle\left[d \ell \partial_{\ell}\right]\left(\frac{G(\lambda) \prod_{s=1}^{n+k-2}\left[a_{s}\left|Q_{i}\right| \ell\right\rangle}{\left\langle\ell\left|K Q_{i}\right| \ell\right\rangle^{n} \prod_{t=1, t \neq i}^{k}\left\langle\ell\left|Q_{t} Q_{i}\right| \ell\right\rangle} \ln \left(-x_{1}^{(i)}\right)\right) .
\end{aligned}
$$

In the last line we made the substitution (4.4).

To complete the calculation for $I_{1}^{(i)}$, we need to take residue of all poles except the one $\left\langle\ell P_{1}^{(i)}\right\rangle$ from factor $\left\langle\ell\left|K Q_{i}\right| \ell\right\rangle$. This seems difficult to do directly. However, notice that the whole expression is holomorphic. Using the result that the sum of residues of all poles of a holomorphic function is zero, we get

$$
I_{1}^{(i)}=-\left.\left(\frac{G(\lambda) \prod_{s=1}^{n+k-2}\left[a_{s}\left|Q_{i}\right| \ell\right\rangle}{\left\langle\ell\left|K Q_{i}\right| \ell\right\rangle^{n} \prod_{t=1, t \neq i}^{k}\left\langle\ell\left|Q_{t} Q_{i}\right| \ell\right\rangle} \ln \left(-x_{1}^{(i)}\right)\right)\right|_{\text {residue of }\left\langle\ell P_{1}^{(i)}\right\rangle}
$$

These residues contribute to triangle coefficients. In the appendix we show how to evaluate the residue of a multiple pole. It can be seen that the expression (4.14) defines the algebraic function for the coefficients with input $a_{i}$ and $Q_{i}$.

Second term of (4.12): The second term of (4.12) is defined by

$$
\begin{aligned}
I_{2}^{(i)} & \equiv \int_{0}^{1} d x \int\langle\ell d \ell\rangle\left[d \ell \partial_{\ell}\right]\left(\frac{F_{i}(\lambda)}{\left\langle\ell\left|Q_{i} K\right| \ell\right\rangle} \frac{\left\langle\ell\left|Q_{i}-K\right| \ell\right]}{x\left\langle\ell\left|Q_{i}-K\right| \ell\right]+\langle\ell|K| \ell]}\right) \\
& =\int_{0}^{1} d x \int\langle\ell d \ell\rangle\left[d \ell \partial_{\ell}\right]\left(-\frac{G(\lambda) \prod_{s=1}^{n+k-2}\left[a_{s}\left|Q_{i}\right| \ell\right\rangle}{\left\langle\ell\left|K Q_{i}\right| \ell\right\rangle^{n} \prod_{t=1, t \neq i}^{k}\left\langle\ell\left|Q_{t} Q_{i}\right| \ell\right\rangle} \frac{\left\langle\ell\left|Q_{i}-K\right| \ell\right]}{x\left\langle\ell\left|Q_{i}-K\right| \ell\right]+\langle\ell|K| \ell]}\right) .
\end{aligned}
$$

Again, we do not take the residue of $\left\langle\ell P_{1}^{(i)}\right\rangle$ from the factor $\left\langle\ell\left|K Q_{i}\right| \ell\right\rangle$.

There are two kinds of poles in (4.16): one is a simple pole from $\left\langle\ell\left|Q_{j} Q_{i}\right| \ell\right\rangle$ and one is a possible multiple pole from $\left\langle\ell P_{2}^{(i)}\right\rangle^{n}$ within $\left\langle\ell\left|K Q_{i}\right| \ell\right\rangle^{n}$. The former contributes to boxes; the latter contributes to triangles. Let us consider the multiple poles first.

When we replace $\left.\mid \ell] \rightarrow \mid P_{2}^{(i)}\right]$, the spinor dependence cancels out in the integrand, giving

$$
\frac{\left\langle\ell\left|Q_{i}-K\right| \ell\right]}{x\left\langle\ell\left|Q_{i}-K\right| \ell\right]+\langle\ell|K| \ell]} \rightarrow \frac{\left(x_{2}^{(i)}+1\right)}{x\left(x_{2}^{(i)}+1\right)-1} .
$$


We integrate over the Feynman parameter and find that the residue is

$$
\left.I_{2}^{(i)}\right|_{\left\langle\ell P_{2}^{(i)}\right\rangle}=\left.\left(-\frac{G(\lambda) \prod_{s=1}^{n+k-2}\left[a_{s}\left|Q_{i}\right| \ell\right\rangle}{\left\langle\ell\left|K Q_{i}\right| \ell\right\rangle^{n} \prod_{t=1, t \neq i}^{k}\left\langle\ell\left|Q_{t} Q_{i}\right| \ell\right\rangle} \ln \left(-x_{2}^{(i)}\right)\right)\right|_{\text {residue of }\left\langle\ell P_{2}^{(i)}\right\rangle} .
$$

Like (4.14), it will contribute to triangles.

Now we move to the simple poles in (4.16). For these simple poles we can evaluate the integral over the Feynman parameter first and get

$$
\left.I_{2}^{(i)}\right|_{\text {simple poles }}=\int\langle\ell d \ell\rangle\left[d \ell \partial_{\ell}\right]\left(\frac{G(\lambda) \prod_{s=1}^{n+k-2}\left[a_{s}\left|Q_{i}\right| \ell\right\rangle}{\left\langle\ell\left|K Q_{i}\right| \ell\right\rangle^{n} \prod_{t=1, t \neq i}^{k}\left\langle\ell\left|Q_{t} Q_{i}\right| \ell\right\rangle} \ln \frac{\langle\ell|K| \ell]}{\left\langle\ell\left|Q_{i}\right| \ell\right]}\right) .
$$

Now we need to compute the residue from the poles in $\left\langle\ell\left|Q_{j} Q_{i}\right| \ell\right\rangle$. Assume without loss of generality that $i<j$, and construct two massless momenta as

$$
P_{1,2}^{(i j)}=Q_{j}+y_{1,2}^{(i j)} Q_{i}, \quad(i<j)
$$

where

$$
y_{1,2}^{(i j)}=\frac{-2 Q_{i} \cdot Q_{j} \pm \sqrt{\Delta^{(i j)}}}{2 Q_{i}^{2}}, \quad \Delta^{(i j)}=\left(2 Q_{i} \cdot Q_{j}\right)^{2}-4 Q_{i}^{2} Q_{j}^{2} .
$$

To simplify our expressions, we define the following function:

$$
F_{i, j}(\ell)=\frac{G(\lambda) \prod_{s=1}^{n+k-2}\left[a_{s}\left|Q_{i}\right| \ell\right\rangle}{\left\langle\ell\left|K Q_{i}\right| \ell\right\rangle^{n} \prod_{t=1, t \neq i, j}^{k}\left\langle\ell\left|Q_{t} Q_{i}\right| \ell\right\rangle} .
$$

To sum the contributions from these two simple poles $\left\langle\ell P_{1}^{(i j)}\right\rangle$ and $\left\langle\ell P_{2}^{(i j)}\right\rangle$, notice that the $P_{1,2}^{(i j)}$ differ only by a sign in front of the square root. Thus we can expand

$$
F_{i, j}\left(P_{1}^{(i j)}\right)=F_{i, j}^{(S)}+F_{i, j}^{(A)}, \quad F_{i, j}\left(P_{2}^{(i j)}\right)=F_{i, j}^{(S)}-F_{i, j}^{(A)} .
$$

Putting this back into the expression (4.18) for the residue, it is straightforward to derive that the contribution from these two poles in $I_{2}^{(i)}$ is

$$
-\frac{1}{\sqrt{\Delta^{(i j)}}}\left(F_{i, j}^{(S)} \ln \frac{\left\langle P_{1}^{(i j)}|K| P_{1}^{(i j)}\right]}{\left\langle P_{1}^{(i j)}\left|Q_{i}\right| P_{1}^{(i j)}\right]} \frac{\left\langle P_{2}^{(i j)}\left|Q_{i}\right| P_{2}^{(i j)}\right]}{\left\langle P_{2}^{(i j)}|K| P_{2}^{(i j)}\right]}+F_{i, j}^{(A)} \ln \frac{\left\langle P_{1}^{(i j)}|K| P_{1}^{(i j)}\right]}{\left\langle P_{1}^{(i j)}\left|Q_{i}\right| P_{1}^{(i j)}\right]} \frac{\left\langle P_{2}^{(i j)}|K| P_{2}^{(i j)}\right]}{\left\langle P_{2}^{(i j)}\left|Q_{i}\right| P_{2}^{(i j)}\right]}\right) .
$$

To proceed further, we notice that the same simple-pole factor $\left\langle\ell\left|Q_{j} Q_{i}\right| \ell\right\rangle$ shows up in the $I^{(j)}$ term (or $F_{j}(\lambda)$ in (4.3)). We can do a similar calculation to get residues in $I_{2}^{(j)}$ in terms the function $F_{j, i}(\lambda)$. One can easily check that $F_{j, i}\left(P_{1,2}^{(i j)}\right)=F_{i, j}\left(P_{1,2}^{(i j)}\right)$, by noticing that

$$
F_{j, i}\left(P_{1,2}^{(i j)}\right)=\frac{G\left(P_{1,2}^{(i j)}\right) \prod_{s=1}^{n+k-2}\left[a_{s} P_{2,1}^{(i j)}\right]}{\left\langle P_{1,2}^{(i j)}|K| P_{2,1}^{(i j)}\right]^{n} \prod_{t=1, t \neq j, i}^{k}\left\langle P_{1,2}^{(i j)}\left|Q_{t}\right| P_{2,1}^{(i j)}\right]}=F_{i, j}\left(P_{1,2}^{(i j)}\right) .
$$


Therefore the sum of contributions from residues associated to $\left\langle\ell\left|Q_{j} Q_{i}\right| \ell\right\rangle=-\left\langle\ell\left|Q_{i} Q_{j}\right| \ell\right\rangle$ in $I_{2}^{(i)}$ and $I_{2}^{(j)}$ is

$$
\begin{aligned}
& -\frac{1}{\sqrt{\Delta^{(i j)}}}\left(F_{i, j}^{(S)} \ln \frac{\left\langle P_{1}^{(i j)}\left|Q_{j}\right| P_{1}^{(i j)}\right]}{\left\langle P_{1}^{(i j)}\left|Q_{i}\right| P_{1}^{(i j)}\right]} \frac{\left\langle P_{2}^{(i j)}\left|Q_{i}\right| P_{2}^{(i j)}\right]}{\left\langle P_{2}^{(i j)}\left|Q_{j}\right| P_{2}^{(i j)}\right]}+F_{i, j}^{(A)} \ln \frac{\left\langle P_{1}^{(i j)}\left|Q_{j}\right| P_{1}^{(i j)}\right]}{\left\langle P_{1}^{(i j)}\left|Q_{i}\right| P_{1}^{(i j)}\right]} \frac{\left\langle P_{2}^{(i j)}\left|Q_{j}\right| P_{2}^{(i j)}\right]}{\left\langle P_{2}^{(i j)}\left|Q_{i}\right| P_{2}^{(i j)}\right]}\right) 4 \\
= & -\frac{1}{\sqrt{\Delta^{(i j)}}}\left(F_{i, j}^{(S)} \ln \frac{y_{1}^{(i j)}}{y_{2}^{(i j)}}+F_{i, j}^{(A)} \ln \frac{Q_{j}^{2}}{Q_{i}^{2}}\right) \\
= & -\frac{1}{\sqrt{\Delta^{(i j)}}}\left(\frac{F_{i, j}\left(P_{1}^{(i j)}\right)+F_{i, j}\left(P_{2}^{(i j)}\right)}{2} \ln \frac{-2 Q_{i} \cdot Q_{j}+\sqrt{\Delta^{(i j)}}}{-2 Q_{i} \cdot Q_{j}-\sqrt{\Delta^{(i j)}}}+\frac{F_{i, j}\left(P_{1}^{(i j)}\right)-F_{i, j}\left(P_{2}^{(i j)}\right)}{2} \ln \frac{Q_{j}^{2} / K^{2}}{Q_{i}^{2} / K^{2}}\right)(.4
\end{aligned}
$$

This is the result we are looking for. The first term is the box contribution. To fix sign conventions, recall that the double cut is given by ${ }^{4}$

$$
\begin{aligned}
& \frac{(1-2 z)}{K^{2}} \int\langle\ell d \ell\rangle[\ell d \ell] \frac{1}{\left\langle\ell\left|Q_{1}\right| \ell\right]\left\langle\ell\left|Q_{2}\right| \ell\right]} \\
& =\frac{(1-2 z)}{K^{2}} \int_{0}^{1} d x \frac{1}{R^{2}}=\frac{(1-2 z)}{K^{2}} \frac{-1}{\sqrt{\Delta^{(i j)}}} \ln \frac{-2 Q_{i} \cdot Q_{j}+\sqrt{\Delta^{(i j)}}}{-2 Q_{i} \cdot Q_{j}-\sqrt{\Delta^{(i j)}}} .
\end{aligned}
$$

Therefore the box coefficient is given by

$$
C_{b o x ; i j}=\frac{K^{2}}{(1-2 z)}\left(\frac{F_{i, j}\left(P_{1}^{(i j)}\right)+F_{i, j}\left(P_{2}^{(i j)}\right)}{2}\right),
$$

where $F_{i, j}$ is given by (4.23).

It is important to realize that in fact (4.27) will be a rational function of $u$ rather than a polynomial, because it contains results for pentagons as well. To separate the box coefficient, we need to write

$$
C_{b o x ; i j}(u)=H(u)+\sum_{i \in \text { pentagons }} A_{i} P_{i},
$$

where $H(u)$ is a polynomial in $u, P_{i}$ is the pentagon cut given by (3.41) and $A_{i}$ is constant in $u$. Thus $H(u)$ will be the true box coefficient, for which we can apply the recursion and reduction formulas, while $A_{i}$ is the true pentagon coefficient.

The second term in (4.26) is the final piece needed for triangles. When we rewrite it as

$$
\begin{aligned}
& -\left.\frac{1}{2} \ln \frac{Q_{i}^{2}}{K^{2}}\left(\frac{G(\lambda) \prod_{s=1}^{n+k-2}\left[a_{s}\left|Q_{i}\right| \ell\right\rangle}{\left\langle\ell\left|K Q_{i}\right| \ell\right\rangle^{n} \prod_{t=1, t \neq i}^{k}\left\langle\ell\left|Q_{t} Q_{i}\right| \ell\right\rangle}\right)\right|_{\text {residue of }\left\langle\ell\left|Q_{j} Q_{i}\right| \ell\right\rangle} \\
& -\left.\frac{1}{2} \ln \frac{Q_{j}^{2}}{K^{2}}\left(\frac{G(\lambda) \prod_{s=1}^{n+k-2}\left[a_{s}\left|Q_{j}\right| \ell\right\rangle}{\left\langle\ell\left|K Q_{j}\right| \ell\right\rangle^{n} \prod_{t=1, t \neq j}^{k}\left\langle\ell\left|Q_{t} Q_{j}\right| \ell\right\rangle}\right)\right|_{\text {residue of }\left\langle\ell\left|Q_{i} Q_{j}\right| \ell\right\rangle},
\end{aligned}
$$

\footnotetext{
${ }^{4}$ In this part, we compare to the case where propagators are massless. For the massive case, we need to adjust the definition of $Q_{i}$ and include a factors of $b$, as explained in section 3 .
} 
we see that the first term is minus half of the residue contribution of $\left\langle\ell\left|Q_{j} Q_{i}\right| \ell\right\rangle$ inside $F_{i}(\lambda)\left(I^{(i)}\right)$ and the second term is minus half of the residue contribution of $\left\langle\ell\left|Q_{i} Q_{j}\right| \ell\right\rangle$ inside $F_{j}(\lambda)\left(I^{(j)}\right)$. For the first term, when we sum up all simple pole contributions for $F_{i}(\lambda)$, we will be left with the residue of the possible multiple pole $\left\langle\ell\left|K Q_{i}\right| \ell\right\rangle^{n}$. That is to say (using the fact that $Q_{i}^{2} / K^{2}=x_{1}^{(i)} x_{2}^{(i)}$ ),

$$
\left.\frac{1}{2} \ln \left(x_{1}^{(i)} x_{2}^{(i)}\right)\left(\frac{G(\lambda) \prod_{s=1}^{n+k-2}\left[a_{s}\left|Q_{i}\right| \ell\right\rangle}{\left\langle\ell\left|K Q_{i}\right| \ell\right\rangle^{n} \prod_{t=1, t \neq i}^{k}\left\langle\ell\left|Q_{t} Q_{i}\right| \ell\right\rangle}\right)\right|_{\text {residue of }\left\langle\ell\left|K Q_{i}\right| \ell\right\rangle} .
$$

Summing the three triangle contributions (4.29) with (4.14) and (4.17) we finally reach

$$
\begin{aligned}
& -\left.\frac{1}{2}\left(\frac{G(\lambda) \prod_{s=1}^{n+k-2}\left[a_{s}\left|Q_{i}\right| \ell\right\rangle}{\left\langle\ell\left|K Q_{i}\right| \ell\right\rangle^{n} \prod_{t=1, t \neq i}^{k}\left\langle\ell\left|Q_{t} Q_{i}\right| \ell\right\rangle} \ln \frac{\left(-x_{1}^{(i)}\right)^{2}}{\left(x_{1}^{(i)} x_{2}^{(i)}\right)}\right)\right|_{\text {residue of }\left\langle\ell P_{1}^{(i)}\right\rangle} \\
& -\left.\frac{1}{2}\left(\frac{G(\lambda) \prod_{s=1}^{n+k-2}\left[a_{s}\left|Q_{i}\right| \ell\right\rangle}{\left\langle\ell\left|K Q_{i}\right| \ell\right\rangle^{n} \prod_{t=1, t \neq i}^{k}\left\langle\ell\left|Q_{t} Q_{i}\right| \ell\right\rangle} \ln \frac{\left(-x_{2}^{(i)}\right)^{2}}{\left(x_{1}^{(i)} x_{2}^{(i)}\right)}\right)\right|_{\text {residue of }\left\langle\ell P_{2}^{(i)}\right\rangle} .
\end{aligned}
$$

Notice that $\ln \left(x_{1}^{(i)} / x_{2}^{(i)}\right)$ is the signature of triangles. To compare, we recall that for triangles we have

$$
(1-2 z) \int\langle\ell d \ell\rangle[\ell d \ell] \frac{1}{\langle\ell|K| \ell]\left\langle\ell\left|Q_{i}\right| \ell\right]}=(1-2 z) \frac{-1}{\sqrt{\Delta^{(i)}}} \ln \frac{-2 Q_{i} \cdot K+\sqrt{\Delta^{(i)}}}{-2 Q_{i} \cdot K-\sqrt{\Delta^{(i)}}} .
$$

Therefore the triangle coefficient is given by

$$
\begin{aligned}
C_{t r i ; i}=\frac{\sqrt{\Delta^{(i)}}}{2(1-2 z)}\{ & \left.\left(\frac{G(\lambda) \prod_{s=1}^{n+k-2}\left[a_{s}\left|Q_{i}\right| \ell\right\rangle}{\left\langle\ell\left|K Q_{i}\right| \ell\right\rangle^{n} \prod_{t=1, t \neq i}^{k}\left\langle\ell\left|Q_{t} Q_{i}\right| \ell\right\rangle}\right)\right|_{\left\langle\ell P_{1}^{(i)}\right\rangle} \\
& \left.-\left.\left(\frac{G(\lambda) \prod_{s=1}^{n+k-2}\left[a_{s}\left|Q_{i}\right| \ell\right\rangle}{\left\langle\ell\left|K Q_{i}\right| \ell\right\rangle^{n} \prod_{t=1, t \neq i}^{k}\left\langle\ell\left|Q_{t} Q_{i}\right| \ell\right\rangle}\right)\right|_{\left\langle\ell P_{2}^{(i)}\right\rangle}\right\} .
\end{aligned}
$$

It is important to observe that both box and triangle coefficients are expressed as the difference of residues at the two poles $P_{1}^{(i)}$ and $P_{2}^{(i)}$. This is the origin of the "signature" square roots of box and triangle integrals. As we will see shortly, for bubble coefficients, we need to add up the residues at the two poles $P_{1}^{(i)}$ and $P_{2}^{(i)}$. The addition will get rid of the square root signature, but we are left with $(1-2 z)$, which is related to bubbles.

\subsection{Rational part}

Now we address bubble coefficients. They are given by the first term of (4.3) with $G_{k}(\lambda)$ given by (4.5). As we have mentioned, for general $n$ and $k$ these are not the simplest and most compact expressions. If we constrain $n \leq 4$, it is possible to use them directly. Here we use a more general approach. 
Our starting point is (4.1). We write it here as

$$
\lim _{\tau \rightarrow 0} \frac{G(\lambda) \prod_{j=1}^{n+k-2}\left[a_{j} \ell\right]}{\prod_{s=0}^{n-1}\langle\ell|K+\tau s \eta| \ell] \prod_{i=1}^{k}\left\langle\ell\left|Q_{i}\right| \ell\right]},
$$

where $\eta^{2}=0$ and $\tau$ is a parameter which we eventually take to zero.

Because no denominator factor appears more than once, we can use (4.7) directly to reach

$$
\begin{aligned}
& \sum_{s=1}^{n-1} \frac{1}{\langle\ell|K| \ell]\langle\ell|K+\tau s \eta| \ell]} \frac{G(\lambda) \prod_{j=1}^{n+k-2}\left[a_{j}|K+\tau s \eta| \ell\right\rangle}{\prod_{s^{\prime}=1, s^{\prime} \neq s}^{n-1}\left\langle\ell\left|\left(K+\tau s^{\prime} \eta\right)(K+\tau s \eta)\right| \ell\right\rangle \prod_{i=1}^{k}\left\langle\ell\left|Q_{i}(K+\tau s \eta)\right| \ell\right\rangle} \\
& +\sum_{i=1}^{k} \frac{1}{\langle\ell|K| \ell]\left\langle\ell\left|Q_{i}\right| \ell\right]} \frac{G(\lambda) \prod_{j=1}^{n+k-2}\left[a_{j}\left|Q_{i}\right| \ell\right\rangle}{\prod_{s^{\prime}=1,}^{n-1}\left\langle\ell\left|\left(K+\tau s^{\prime} \eta\right) Q_{i}\right| \ell\right\rangle \prod_{i^{\prime}=1, i^{\prime} \neq i}^{k}\left\langle\ell\left|Q_{i^{\prime}} Q_{i}\right| \ell\right\rangle} .
\end{aligned}
$$

When we take the $\tau \rightarrow 0$ limit, the second line becomes the contribution to triangles and boxes that was discussed in the previous subsection. The first line gives the bubble contribution. It appears that each term in the first line diverges in the $\tau \rightarrow 0$ limit. To proceed, define

$$
\widetilde{K}(s)=K+\tau s \eta \text {. }
$$

Then we find that each term in the first line of $(4.33)$ can be rewritten as

$$
\frac{1}{\langle\ell|\widetilde{K}(s)-\tau s \eta| \ell]\langle\ell|\widetilde{K}(s)| \ell]} \frac{G(\lambda) \prod_{j=1}^{n+k-2}\left[a_{j}|\widetilde{K}(s)| \ell\right\rangle}{\tau^{n-2}\langle\ell|\eta \widetilde{K}(s)| \ell\rangle^{n-2} \prod_{s^{\prime}=1, s^{\prime} \neq s}^{n-1}\left(s^{\prime}-s\right) \prod_{i=1}^{k}\left\langle\ell\left|Q_{i} \widetilde{K}(s)\right| \ell\right\rangle} .
$$

Now we can expand this expression as a power series in $\tau$, keeping those terms that will survive the limit $\tau \rightarrow 0$. Specifically, we substitute

$$
\frac{1}{\langle\ell|\widetilde{K}(s)-\tau s \eta| \ell]\langle\ell|\widetilde{K}(s)| \ell]}=\sum_{h=0}^{n-2} \frac{\tau^{h} s^{h}\langle\ell|\eta| \ell]^{h}}{\langle\ell|\widetilde{K}(s)| \ell]^{2+h}}+\mathcal{O}\left(\tau^{n-1}\right) .
$$

Apply the familiar spinor integration procedure to replace one integrand by a total derivative using the identity

$$
[\ell d \ell]\left(\frac{[\eta \ell]^{n}}{\langle\ell|P| \ell]^{n+2}}\right)=\left[d \ell \partial_{\ell}\right]\left(\frac{1}{(n+1)} \frac{1}{\langle\ell|P| \eta]} \frac{[\eta \ell]^{n+1}}{\langle\ell|P| \ell]^{n+1}}\right) .
$$

The result is then the sum of residues of

$$
\frac{G(\lambda) \prod_{j=1}^{n+k-2}\left[a_{j}|\widetilde{K}(s)| \ell\right\rangle}{\tau^{n-2}\langle\ell|\eta \widetilde{K}(s)| \ell\rangle^{n-1} \prod_{s^{\prime}=1, s^{\prime} \neq s}^{n-1}\left(s^{\prime}-s\right) \prod_{i=1}^{k}\left\langle\ell\left|Q_{i} \widetilde{K}(s)\right| \ell\right\rangle}\left(\sum_{h=0}^{n-2} \frac{\tau^{h} s^{h}\langle\ell|\eta| \ell]^{h+1}}{(h+1)\langle\ell|\widetilde{K}(s)| \ell]^{1+h}}\right) .
$$

For the multiple pole, $\langle\ell|\eta \widetilde{K}(s)| \ell\rangle=\langle\ell \eta\rangle[\eta|\widetilde{K}(s)| \ell\rangle$. Since the numerator contains a factor of $[\eta \ell]^{h+1}$, we do not take the residue of $\langle\ell \eta\rangle$. 
Finally, after defining the quantity

$$
\begin{aligned}
R\left[\widetilde{K}(s),\left\{Q_{i}\right\}, \eta\right]= & \sum_{\text {poles except } \eta} \operatorname{Res}\left(\frac{G(\lambda) \prod_{j=1}^{n+k-2}\left[a_{j}|\widetilde{K}(s)| \ell\right\rangle}{\tau^{n-2}\langle\ell|\eta \widetilde{K}(s)| \ell\rangle^{n-1} \prod_{s^{\prime}=1, s^{\prime} \neq s}^{n-1}\left(s^{\prime}-s\right) \prod_{i=1}^{k}\left\langle\ell\left|Q_{i} \widetilde{K}(s)\right| \ell\right\rangle}\right. \\
& \left.\times\left(\sum_{h=0}^{n-2} \frac{\tau^{h} s^{h}\langle\ell|\eta| \ell]^{h+1}}{(h+1)\langle\ell|\widetilde{K}(s)| \ell]^{1+h}}\right)\right),
\end{aligned}
$$

we can write the bubble coefficients as

$$
C_{\text {bubble }}=\left.\frac{1}{\sqrt{1-u}} \sum_{s=1}^{n-1} R\left[\widetilde{K}(s),\left\{Q_{i}\right\}, \eta\right]\right|_{\tau \rightarrow 0} .
$$

The limit is taken by expanding and truncating the series.

\subsection{Summary of results}

Conventions:

We start from an integrand of the form

$$
I_{\text {term }}=\frac{G(\lambda) \prod_{j=1}^{n+k-2}\left[a_{j} \ell\right]}{\langle\ell|K| \ell]^{n} \prod_{i=1}^{k}\left\langle\ell\left|Q_{i}\right| \ell\right]}
$$

and present here an expression to be integrated over the final $(-2 \epsilon)$ dimensions as discussed in Section 2.3.

Box coefficients:

$$
\begin{gathered}
C_{b o x ; i j}=\frac{K^{2}}{\sqrt{1-u}}\left(\frac{F_{i, j}\left(P_{1}^{(i j)}\right)+F_{i, j}\left(P_{2}^{(i j)}\right)}{2}\right) \\
P_{1,2}^{(i j)}=Q_{j}+y_{1,2}^{(i j)} Q_{i} \quad(i<j) \\
y_{1,2}^{(i j)}=\frac{-2 Q_{i} \cdot Q_{j} \pm \sqrt{\Delta^{(i j)}}}{2 Q_{i}^{2}}, \quad \Delta^{(i j)}=\left(2 Q_{i} \cdot Q_{j}\right)^{2}-4 Q_{i}^{2} Q_{j}^{2} \\
F_{i, j}(\ell)=\frac{G(\lambda) \prod_{s=1}^{n+k-2}\left[a_{s}\left|Q_{i}\right| \ell\right\rangle}{\left\langle\ell\left|K Q_{i}\right| \ell\right\rangle^{n} \prod_{t=1, t \neq i, j}^{k}\left\langle\ell\left|Q_{t} Q_{i}\right| \ell\right\rangle}
\end{gathered}
$$

Triangle coefficients: 


$$
\begin{gathered}
C_{t r i ; i}=\frac{\sqrt{\Delta^{(i)}}}{2 \sqrt{1-u}}\left\{\left.\left(\frac{G(\lambda) \prod_{s=1}^{n+k-2}\left[a_{s}\left|Q_{i}\right| \ell\right\rangle}{\left\langle\ell\left|K Q_{i}\right| \ell\right\rangle^{n} \prod_{t=1, t \neq i}^{k}\left\langle\ell\left|Q_{t} Q_{i}\right| \ell\right\rangle}\right)\right|_{\left\langle\ell P_{1}^{(i)}\right\rangle}\right. \\
\left.-\left.\left(\frac{G(\lambda) \prod_{s=1}^{n+k-2}\left[a_{s}\left|Q_{i}\right| \ell\right\rangle}{\left\langle\ell\left|K Q_{i}\right| \ell\right\rangle^{n} \prod_{t=1, t \neq i}^{k}\left\langle\ell\left|Q_{t} Q_{i}\right| \ell\right\rangle}\right)\right|_{\left\langle\ell P_{2}^{(i)}\right\rangle}\right\} \\
P_{1,2}^{(i)}=Q_{i}+x_{1,2}^{(i)} K \\
x_{1,2}^{(i)}=\frac{-2 Q_{i} \cdot K \pm \sqrt{\Delta^{(i)}}}{2 K^{2}} \quad \Delta^{(i)}=\left(2 Q_{i} \cdot K\right)^{2}-4 Q_{i}^{2} K^{2}
\end{gathered}
$$

Bubble coefficients:

$$
C_{b u b b l e}=\left.\frac{1}{\sqrt{1-u}} \sum_{s=1}^{n-1} R\left[\widetilde{K}(s),\left\{Q_{i}\right\}, \eta\right]\right|_{\tau \rightarrow 0}
$$

The limit is taken by expanding and truncating the series.

$$
\begin{aligned}
& R\left[\widetilde{K}(s),\left\{Q_{i}\right\}, \eta\right]= \sum_{\text {poles except } \eta} \operatorname{Res}\left(\frac{G(\lambda) \prod_{j=1}^{n+k-2}\left[a_{j}|\widetilde{K}(s)| \ell\right\rangle}{\tau^{n-2}\langle\ell|\eta \widetilde{K}(s)| \ell\rangle^{n-1} \prod_{s^{\prime}=1, s^{\prime} \neq s}^{n-1}\left(s^{\prime}-s\right) \prod_{i=1}^{k}\left\langle\ell\left|Q_{i} \widetilde{K}(s)\right| \ell\right\rangle}\right. \\
&\left.\times\left(\sum_{h=0}^{n-2} \frac{\tau^{h} s^{h}\langle\ell|\eta| \ell]^{h+1}}{(h+1)\langle\ell|\widetilde{K}(s)| \ell]^{1+h}}\right)\right) \\
& \widetilde{K}(s)=K+\tau s \eta
\end{aligned}
$$

For a detailed description of evaluation, see the appendix.

\section{Discussion}

In this section, we first comment on the input needed for this integration program, and then discuss

the alternative approaches of generalized unitarity cuts and the program of Ossola, Papadopoulos and Pittau [2]. 


\subsection{Tree level input}

The input needed for the unitary cut method is a collection of (on-shell) tree-level amplitudes, which can be calculated by recursion relations. However, results with spurious poles (which generally arise in the most compact expressions) can make it hard to apply our method. To avoid this difficulty, we can apply spinor identities to regroup the terms into an expression free of spurious poles.

Let us demonstrate this in one example. The five point function with two massive scalars are given by $^{5}$

$$
\begin{aligned}
A\left(\ell_{1}, 1^{+}, 2^{+}, 3^{-}, \ell_{2}\right)= & -\frac{\left\langle 3\left|\ell_{2}(1+2) \ell_{1}\right| 1\right]^{2}}{\langle 12\rangle\langle 23\rangle\left(\left(\ell_{1}+k_{1}\right)^{2}-\mu^{2}\right)\left(\left(\ell_{2}+k_{3}\right)^{2}-\mu^{2}\right)\left[3\left|(1+2) \ell_{1}\right| 1\right]} \\
& +\frac{\mu^{2}[12]^{3}}{K_{123}^{2}[23]\left[3\left|(1+2) \ell_{1}\right| 1\right]} \\
= & -\frac{\left\langle 3\left|\ell_{2}(1+2) \ell_{1}\right| 1\right]^{2}}{\langle 12\rangle\langle 23\rangle\left\langle 1\left|\ell_{1}\right| 1\right]\left\langle 3\left|\ell_{2}\right| 3\right]\left[3\left|(1+2) \ell_{1}\right| 1\right]} \\
& +\frac{\mu^{2}[12]^{3}}{K_{123}^{2}[23]\left[3\left|(1+2) \ell_{1}\right| 1\right]} .
\end{aligned}
$$

The spurious pole in the first term can be split from the others by the identity

$$
\frac{\left.\left\langle 3\left|\ell_{2}(1+2)\right| \ell_{1}\right| 1\right]}{\left\langle 1\left|\ell_{1}\right| 1\right]\left[3|(1+2)| \ell_{1} \mid 1\right]}=\frac{\left\langle 3\left|\ell_{2}(1+2)\right| 1\right\rangle}{[3|(1+2)| 1\rangle\left\langle 1\left|\ell_{1}\right| 1\right]}+\frac{\left\langle 3\left|\ell_{2}(1+2)(1+2)\right| 3\right]}{\langle 1|(1+2)| 3]\left[3|(1+2)| \ell_{1} \mid 1\right]}
$$

Then

$$
\begin{aligned}
A\left(\ell_{1}, 1^{+}, 2^{+}, 3^{-}, \ell_{2}\right)= & -\frac{\left\langle 3\left|\ell_{2}(1+2) \ell_{1}\right| 1\right]\left\langle 3\left|\ell_{2}\right| 2\right]}{\langle 12\rangle\langle 23\rangle[32]\left\langle 3\left|\ell_{2}\right| 3\right]\left\langle 1\left|\ell_{1}\right| 1\right]}+\frac{\left\langle 3\left|\ell_{2}(1+2) \ell_{1}\right| 1\right][12]}{\langle 12\rangle\langle 23\rangle[23]\left[3\left|(1+2) \ell_{1}\right| 1\right]} \\
& +\frac{\mu^{2}[12]^{3}}{K_{123}^{2}[23]\left[3\left|(1+2) \ell_{1}\right| 1\right]} .
\end{aligned}
$$

Using

$$
\begin{aligned}
\left\langle 3\left|\ell_{2}(1+2) \ell_{1}\right| 1\right] K_{123}^{2} & =\left\langle 3\left|\ell_{2}(1+2) \ell_{1}\right| 1\right]\left(K_{12}^{2}-\langle 3|(1+2)| 3]\right) \\
& =\left\langle 3\left|\ell_{2}(1+2) \ell_{1}\right| 1\right] K_{12}^{2}+\left\langle 3\left|(1+2) \ell_{2}\right| 3\right]\left[3\left|(1+2) \ell_{1}\right| 1\right]+K_{12}^{2}\left\langle 3\left|\ell_{1}\right| 1\right]\left\langle 3\left|\ell_{2}\right| 3\right],
\end{aligned}
$$

we can add the last two terms together and see that the spurious pole $\left[3\left|(1+2) \ell_{1}\right| 1\right]$ has been canceled (as it must). Thus we finally get

$$
A\left(\ell_{1}, 1^{+}, 2^{+}, 3^{-}, \ell_{2}\right)=\frac{\left\langle 3\left|\ell_{2}(1+2) \ell_{1}\right| 1\right]\left\langle 3\left|\ell_{2}\right| 2\right]}{\langle 12\rangle K_{23}^{2}\left\langle 1\left|\ell_{1}\right| 1\right]\left\langle 3\left|\ell_{2}\right| 3\right]}+\frac{[12]\left\langle 3\left|(1+2) \ell_{2}\right| 3\right\rangle}{\langle 12\rangle K_{23}^{2} K_{123}^{2}} .
$$

A similar calculation gives

$$
A_{5}\left(\ell_{1}^{+}, 1^{+}, 2^{-}, 3^{+}, \ell_{2}^{-}\right)=\frac{\left\langle 2\left|\ell_{1}\right| 1\right]\left\langle 2\left|\ell_{2}\right| 3\right]^{2}}{\langle 12\rangle K_{23}^{2}\left\langle 3\left|\ell_{2}\right| 3\right]\left\langle 1\left|\ell_{1}\right| 1\right]}+\frac{[31]\left\langle 2\left|\ell_{1}\right| 1\right]\left\langle 2\left|\ell_{2}\right| 3\right]}{K_{12}^{2} K_{23}^{2}\left\langle 3\left|\ell_{2}\right| 3\right]}-\frac{\left\langle 2\left|\ell_{2} K_{123}\right| 2\right\rangle[13]^{2}}{K_{12}^{2} K_{23}^{2} K_{123}^{2}}
$$

where spurious poles have been canceled.

\footnotetext{
${ }^{5}$ The sign of the second term is different from the corresponding formula in 24. We have checked, using the method of 24], that the sign in this earlier formula was a typo.
} 


\subsection{The quadruple cut}

In four dimensions, the quadruple cut [5] is a powerful tool for getting box coefficients because the integral is completely localized. Quadruple cuts may be applied here as well since we have separated the $(-2 \epsilon)$-dimensional and four-dimensional integrations. We apply them to boxes and pentagons.

Again, we write in the language of spinor integration (see the brief remarks at the beginning of Section 3 ), but this is absolutely not essential to the technique of quadruple cuts.

Let us start with the quadruple cut (denoted here by $C^{q}$ ) of a box. It is given by

$$
\begin{aligned}
C_{4}^{q}= & \int_{0}^{1} d u u^{-1-\epsilon} \int d^{4} \ell \delta^{+}\left(\ell^{2}\right) \delta\left((1-2 z) K^{2}-2 \ell \cdot K+M_{1}^{2}-M_{2}^{2}\right) \\
& \delta\left(\left(\widetilde{\ell}-P_{1}\right)^{2}-M_{3}^{2}-\mu^{2}\right) \delta\left(\left(\widetilde{\ell}-P_{2}\right)^{2}-M_{4}^{2}-\mu^{2}\right) \\
= & \int_{0}^{1} d u u^{-1-\epsilon} \int d^{4} \ell \delta^{+}\left(\ell^{2}\right) \delta\left((1-2 z) K^{2}-2 \ell \cdot K+M_{1}^{2}-M_{2}^{2}\right) \\
& \delta\left(P_{1}^{2}+M_{1}^{2}-M_{3}^{2}-z\left(2 K \cdot P_{1}\right)-2 \ell \cdot P_{1}\right) \delta\left(P_{2}^{2}+M_{1}^{2}-M_{4}^{2}-z\left(2 K \cdot P_{2}\right)-2 \ell \cdot P_{2}\right) .
\end{aligned}
$$

After $t$-integration, which sets $t=-\left((1-2 z) K^{2}+M_{1}^{2}-M_{2}^{2}\right) /\langle\ell|K| \ell]$, we have

$$
C_{4}^{q}=\int_{0}^{1} d u u^{-1-\epsilon} \int\langle\ell d \ell\rangle[\ell d \ell] \frac{(1-2 z) K^{2}+M_{1}^{2}-M_{2}^{2}}{\langle\ell|K| \ell]^{2}} \delta\left(\frac{K^{2}\left\langle\ell\left|Q_{1}\right| \ell\right]}{\langle\ell|K| \ell]}\right) \delta\left(\frac{K^{2}\left\langle\ell\left|Q_{2}\right| \ell\right]}{\langle\ell|K| \ell]}\right),
$$

where

$$
Q_{i}=-\left((1-2 z)+\frac{M_{1}^{2}-M_{2}^{2}}{K^{2}}\right) P_{1}+\frac{P_{1}^{2}+M_{1}^{2}-M_{i+2}^{2}-z\left(2 K \cdot P_{1}\right)}{K^{2}} K
$$

To solve the remaining two delta-functions, we need to find two momenta constructed from $q_{i}=Q_{2}+x_{i} Q_{1}$, $i=1,2$ such that $q_{i}^{2}=0$. This is the same construction as in (4.20). Then the two solutions for the two delta-functions are given by ${ }^{6}$

$$
\left.\left.\ell_{1}=\left|q_{1}\right\rangle \mid q_{2}\right], \quad \ell_{2}=\left|q_{2}\right\rangle \mid q_{1}\right]
$$

Notice that these solutions are complex, as usual for quadruple cuts. There is a universal Jacobian factor, which in general is a function of $u$. Putting it all together we have

$$
C_{4}^{q}=\int_{0}^{1} d u u^{-1-\epsilon} J(u)
$$

where $J(u)$ is the Jacobian and should be symmetric in $K, P_{1}, P_{2}$.

Next we consider the quadruple cut of pentagons. The calculation is similar, so we shall be brief. After $t$-integration we have

$$
C_{5}^{q}=\int_{0}^{1} d u u^{-1-\epsilon} \int\langle\ell d \ell\rangle[\ell d \ell] \frac{(1-2 z) K^{2}+M_{1}^{2}-M_{2}^{2}}{\langle\ell|K| \ell] K^{2}\left\langle\ell\left|Q_{3}\right| \ell\right]} \delta\left(\frac{K^{2}\left\langle\ell\left|Q_{1}\right| \ell\right]}{\langle\ell|K| \ell]}\right) \delta\left(\frac{K^{2}\left\langle\ell\left|Q_{2}\right| \ell\right]}{\langle\ell|K| \ell]}\right),
$$

\footnotetext{
${ }^{6}$ These are the $\ell_{3}, \ell_{4}$ of $[$.
} 
with the $Q_{i}$ defined in $(5.3)$.

Now we need to sum up the contributions of the two solutions. The Jacobian of the two solutions is the same. We end up with ${ }^{7}$

$$
C_{5}^{q}=\int_{0}^{1} d u u^{-1-\epsilon} \frac{J(u)}{K^{2}} \frac{1}{2}\left(\frac{\left\langle q_{1}|K| q_{2}\right]}{\left\langle q_{1}\left|Q_{3}\right| q_{2}\right]}+\frac{\left\langle q_{2}|K| q_{1}\right]}{\left\langle q_{2}\left|Q_{3}\right| q_{1}\right]}\right)=\int_{0}^{1} d u u^{-1-\epsilon} \frac{J(u)}{2 K^{2}} S\left[Q_{2}, Q_{1}, Q_{3}, K\right],
$$

where $S\left[Q_{2}, Q_{1}, Q_{3}, K\right]$ was defined in (3.42.) We see again that the relative factor between boxes and pentagons is $S[\bullet] /\left(2 K^{2}\right)$, just as we learned from the double cut.

In general, we perform the $t$-integral first and then add the two solutions. This gives a rational function $R(u)$. Then we need to split

$$
R(u)=P(u)+\sum_{Q_{3}} a_{Q_{3}} \frac{S\left[Q_{2}, Q_{1}, Q_{3}, K\right]}{2 K^{2}},
$$

where $P(u)$ is a polynomial that gives the box contribution, and $a_{Q_{3}}$ are constants in $u$ that give corresponding coefficients of pentagons. This decomposition is the same as the one given by (4.28).

After getting box and pentagon coefficients from quadruple cuts, one might continue by applying triple cuts to target specific triangle coefficients, and then finally use the usual double cut for the bubble part only. Triple cuts have been used to get particular one-loop coefficients in [25, 26, 27, 28], and recently a nice paper [11] has described a general procedure to compute triple cuts in arbitrary dimensions. One can try to systematically study the triple cuts of [1] along the lines presented in this paper. It is easy to see that the delta-function there (plus possible derivatives of delta-function) corresponds to our multiple pole.

\subsection{Comparison with OPP method}

Recently, a computation by Ossola, Papadopoulos and Pittau (OPP) [2] has been attracting attention. It seems to be a very simple reduction method, which can be performed at the integrand level. The key point of the OPP method is that knowing the general form of spurious terms, one can solve algebraically for the coefficients of physical and spurious terms from knowing the initial data. The work we have presented here is in the same spirit. In our method, it is through splitting into partial fractions that we are able to identify contributions to the various basis integrals and find the functions for these coefficients.

We remark that in [2], it is claimed that in the renormalizable gauge, there is an upper bound on the number of spurious terms. For example, there are six for triangles, eight for bubbles. The upper bound in the OPP method will correspond to the upper bound of $n$ in our factor $\langle\ell|K| \ell]^{n}$. Our experience suggests that we will always find $n \leq 4$. The reason is the following. Before the $t$-integration we have

$$
\frac{\prod_{i=1}^{N_{1}}\left[a_{i}|\widetilde{\ell}| b_{i}\right\rangle}{\prod_{j=1}^{N_{2}}\left(\left(\widetilde{\ell}-P_{j}\right)^{2}-\mu^{2}\right)}
$$

\footnotetext{
${ }^{7}$ Here we have inserted the factor $1 / 2$ since we need to sum the contributions of two solutions. This is the same expression used in original quadruple cut 5], where on one side, we sum up two solutions and divide by two, while on the other side, we have just the Jacobi factor for the basis of box.
} 
where the power of $\tilde{\ell}$ in the numerator $N_{1}$ has an upper bound, $N_{1} \leq N_{2}+2$. The reason is that in renormalizable gauge, the expression derived from Feynman rules has the property that the degree of $\widetilde{\ell}$ in numerator is less than or equal to that in the denominator. After peeling off the two cut propagators, we have $N_{1} \leq N_{2}+2$. Now we count the powers of $1 /\langle\ell|K| \ell]$ after $t$-integration. Numerators contribute $-N_{1}$ while denominators contribute $N_{2}$. From $\int t d t$ and the delta-function, we get an additional -2 . In all, we have $(1 /\langle\ell|K| \ell])^{-N_{1}-2+N_{2}}$, thus $n=N_{1}+2-N_{2} \leq 4$.

Assuming that indeed $n \leq 4$, the correspondence between our method and the OPP method becomes clearer. It is easy to see that box and pentagon coefficients have the same expressions. For triangle, we have multiple poles up to order 4. From our discussion in the Appendix, it can be seen that a pole of order $n$ can be traded for a simple pole with an $(n-1)$-th derivative. In our case, with $n \leq 4$ we have up to third derivatives. This is similar to $j=1,2,3$ in [2]. For bubble coefficients, we have up to three terms, and each one has multiple poles up to order three, so we would expect $3 \times 2=6$ contributions, compared to the eight spurious terms of the OPP method. Our impression is that the spurious terms of OPP are hidden as multiple poles as well as simple poles in our language. The algebraic complexity of the two methods should be equivalent.

\section{Acknowledgments}

We wish to thank C. Anastasiou for helpful discussions and feedback on the manuscript and R. Schabinger for informing us of several typos in earlier versions. RB is supported by Stichting FOM. BF is supported by the Marie-Curie Research Training Network under contract MRTN-CT-2004-005104.

\section{A. Evaluation of residues}

In this appendix, we discuss how to evaluate the various functions we defined in section 4 to get formulas for coefficients of the basis integrals. Our aim is to make the formulas programmable, so we go into some detail.

\section{A.1 Box coefficients}

The box (and pentagon) coefficients are given by (4.27),

$$
C_{b o x ; i j}=\frac{K^{2}}{(1-2 z)}\left(\frac{F_{i, j}\left(P_{1}^{(i j)}\right)+F_{i, j}\left(P_{2}^{(i j)}\right)}{2}\right),
$$

where

$$
F_{i, j}\left(P_{1,2}^{(i j)}\right)=\frac{G\left(P_{1,2}^{(i j)}\right) \prod_{s=1}^{n+k-2}\left[a_{s} P_{2,1}^{(i j)}\right]}{\left\langle P_{1,2}^{(i j)}|K| P_{2,1}^{(i j)}\right]^{n} \prod_{t=1, t \neq j, i}^{k}\left\langle P_{1,2}^{(i j)}\left|Q_{t}\right| P_{2,1}^{(i j)}\right]}
$$


Let us rewrite the coefficient as

$$
C_{b o x ; i j}=\frac{K^{2}}{2(1-2 z)} \frac{N}{D}
$$

where $^{8}$

$$
\begin{aligned}
\frac{N}{D}= & F_{i, j}\left(P_{1}^{(i j)}\right)+F_{i, j}\left(P_{2}^{(i j)}\right) \\
N= & G\left(P_{1}^{(i j)}\right) \prod_{s=1}^{n+k-2}\left[a_{s} P_{2}^{(i j)}\right]\left\langle P_{2}^{(i j)}|K| P_{1}^{(i j)}\right]^{n} \prod_{t=1, t \neq j, i}^{k}\left\langle P_{2}^{(i j)}\left|Q_{t}\right| P_{1}^{(i j)}\right] \\
& +G\left(P_{2}^{(i j)}\right) \prod_{s=1}^{n+k-2}\left[a_{s} P_{1}^{(i j)}\right]\left\langle P_{1}^{(i j)}|K| P_{2}^{(i j)}\right]^{n} \prod_{t=1, t \neq j, i}^{k}\left\langle P_{1}^{(i j)}\left|Q_{t}\right| P_{2}^{(i j)}\right] \\
& \quad\left\langle P_{1}^{(i j)}|K| P_{2}^{(i j)}\right]^{n} \prod_{t=1, t \neq j, i}^{k}\left\langle P_{1}^{(i j)}\left|Q_{t}\right| P_{2}^{(i j)}\right]\left\langle P_{2}^{(i j)}|K| P_{1}^{(i j)}\right]^{n} \prod_{t=1, t \neq j, i}^{k}\left\langle P_{2}^{(i j)}\left|Q_{t}\right| P_{1}^{(i j)}\right] .
\end{aligned}
$$

For $D$ we use the following identity:

$$
\left\langle\eta_{1}|S| \eta_{2}\right]\left\langle\eta_{2}|S| \eta_{1}\right]=\left(2 \eta_{1} \cdot S\right)\left(2 \eta_{2} \cdot S\right)-S^{2}\left(2 \eta_{1} \cdot \eta_{2}\right) .
$$

Then,

$$
\begin{aligned}
\left\langle P_{1}^{(i j)}|K| P_{2}^{(i j)}\right]\left\langle P_{2}^{(i j)}|K| P_{1}^{(i j)}\right] & =\left(2 K \cdot\left(Q_{j}+y_{1}^{(i j)} Q_{i}\right)\right)\left(2 K \cdot\left(Q_{j}+y_{1}^{(i j)} Q_{i}\right)\right)+\frac{K^{2} \Delta^{(i j)}}{Q_{i}^{2}} \\
& \equiv T\left[Q_{i}, Q_{j}, K\right] .
\end{aligned}
$$

The function $T\left[Q_{i}, Q_{j}, K\right]$ in this formula is $T_{2} / Q_{i}^{2}$, with the definition of $T_{2}$ given in (3.43). Using this definition we have

$$
D=T\left[Q_{i}, Q_{j}, K\right]^{n} \prod_{t=1, t \neq j, i}^{k} T\left[Q_{i}, Q_{j}, Q_{t}\right]
$$

It is easy to see that each term in $N$ may be written as a product of the form

$$
\left[\begin{array}{ll}
a_{s} & P_{2}
\end{array}\right]\left\langle P_{2}|Q| P_{1}\right]\left\langle P_{1} b_{s}\right\rangle=\left[a_{s}\left|P_{2} Q P_{1}\right| b_{s}\right\rangle=\left[a_{s}\left|\left(Q_{j}+y_{2} Q_{i}\right) Q\left(Q_{j}+y_{1} Q_{i}\right)\right| b_{s}\right\rangle .
$$

Thus we see that the evaluation of box coefficients (A.2) is straightforward.

\section{A.2 The residue of multiple poles}

For triangles and bubbles, we need to know how to get residue of multiple poles. There are several ways to do this. The first one is to use the splitting method discussed in [7].

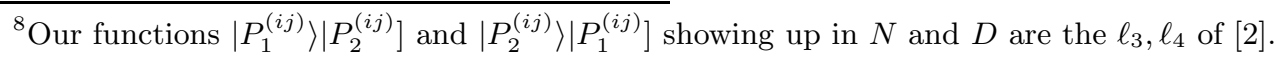


The second method is to shift momentum parametrically and then take the limit where the parameter goes to zero. We illustrate this method with the example of a double pole. We denote the small parameter by $\tau$.

$$
\begin{aligned}
\frac{1}{\langle\ell \eta\rangle^{2}} \frac{\prod_{j=1}^{k}\left\langle\ell a_{j}\right\rangle}{\prod_{j=1}^{k}\left\langle\ell b_{j}\right\rangle} & \rightarrow \frac{1}{\langle\ell \eta\rangle\langle\ell(\eta+\tau \alpha)\rangle} \frac{\prod_{j=1}^{k}\left\langle\ell a_{j}\right\rangle}{\prod_{j=1}^{k}\left\langle\ell b_{j}\right\rangle} \\
& =\frac{1}{\langle\eta(\eta+\tau \alpha)\rangle} \frac{\prod_{j=1}^{k}\left\langle\eta a_{j}\right\rangle}{\prod_{j=1}^{k}\left\langle\eta b_{j}\right\rangle}-\frac{1}{\langle\eta(\eta+\tau \alpha)\rangle} \frac{\prod_{j=1}^{k}\left\langle(\eta+\tau \alpha) a_{j}\right\rangle}{\prod_{j=1}^{k}\left\langle(\eta+\tau \alpha) b_{j}\right\rangle} \\
& =\frac{1}{\tau\langle\eta \alpha\rangle} \frac{\prod_{j=1}^{k}\left\langle\eta a_{j}\right\rangle}{\prod_{j=1}^{k}\left\langle\eta b_{j}\right\rangle}\left(1-\frac{\prod_{j=1}^{k}\left(1+\tau \frac{\left\langle\alpha a_{j}\right\rangle}{\left\langle\eta a_{j}\right\rangle}\right)}{\prod_{j=1}^{k}\left(1+\tau \frac{\left\langle\alpha b_{j}\right\rangle}{\left\langle\eta b_{j}\right\rangle}\right)}\right) \\
& \rightarrow \frac{1}{\langle\eta \alpha\rangle} \frac{\prod_{j=1}^{k}\left\langle\eta a_{j}\right\rangle}{\prod_{j=1}^{k}\left\langle\eta b_{j}\right\rangle}\left(-\sum_{j=1}^{k} \frac{\left\langle\alpha a_{j}\right\rangle}{\left\langle\eta a_{j}\right\rangle}+\sum_{j=1}^{k} \frac{\left\langle\alpha b_{j}\right\rangle}{\left\langle\eta b_{j}\right\rangle}\right) \\
& =\frac{\prod_{j=1}^{k}\left\langle\eta a_{j}\right\rangle}{\prod_{j=1}^{k}\left\langle\eta b_{j}\right\rangle}\left(\sum_{j=1}^{k} \frac{\left\langle a_{j} b_{j}\right\rangle}{\left\langle\eta a_{j}\right\rangle\left\langle\eta b_{j}\right\rangle}\right) .
\end{aligned}
$$

We see that this is the correct result. Compared to the first method, we have more terms and one extra auxiliary spinor $\eta$. However, in this method, the symmetry among $a_{i}$ 's and $b_{i}$ 's is explicit.

Now we can use the above idea to get the general expression for multiple poles:

$$
\begin{aligned}
\frac{1}{\langle\ell \eta\rangle^{n}} \frac{\prod_{i}\left\langle\ell a_{i}\right\rangle}{\prod_{j}\left\langle\ell b_{j}\right\rangle} & \rightarrow \frac{1}{\prod_{s=0}^{n-1}\langle\ell(\eta+s \tau \alpha)\rangle} \frac{\prod_{i}\left\langle\ell a_{i}\right\rangle}{\prod_{j}\left\langle\ell b_{j}\right\rangle} \\
& =\sum_{s_{0}=0}^{n-1} \frac{1}{\prod_{s=0, s \neq s_{0}}^{n-1}\left\langle\left(\eta+\tau s_{0} \alpha\right)(\eta+s \tau \alpha)\right\rangle} \frac{\prod_{i}\left\langle\left(\eta+\tau s_{0} \alpha\right) a_{i}\right\rangle}{\prod_{j}\left\langle\left(\eta+\tau s_{0} \alpha\right) b_{j}\right\rangle} \\
& =\sum_{s_{0}=0}^{n-1} \frac{1}{\tau^{n-1}\langle\eta \alpha\rangle^{n-1} \prod_{s=0, s \neq s_{0}}^{n-1}\left(s-s_{0}\right)} \frac{\prod_{i}\left\langle\left(\eta+\tau s_{0} \alpha\right) a_{i}\right\rangle}{\prod_{j}\left\langle\left(\eta+\tau s_{0} \alpha\right) b_{j}\right\rangle} \\
& =\sum_{s_{0}=0}^{n-1} \frac{1}{\tau^{n-1}\langle\eta \alpha\rangle^{n-1} \prod_{s=0, s \neq s_{0}}^{n-1}\left(s-s_{0}\right)} \frac{\prod_{i}\left\langle\eta a_{i}\right\rangle\left(1+\tau s_{0} \frac{\left\langle\alpha a_{i}\right\rangle}{\left\langle\eta a_{i}\right\rangle}\right)}{\prod_{j}\left\langle\eta b_{j}\right\rangle\left(1+\tau s_{0} \frac{\left\langle\alpha b_{j}\right\rangle}{\left\langle\eta b_{j}\right\rangle}\right)} \\
& =\frac{1}{\prod^{n-1}\langle\eta \alpha\rangle^{n-1}} \frac{\left.1+\tau s_{0} \frac{\left\langle\alpha a_{i}\right\rangle}{\left\langle\eta a_{i}\right\rangle}\right)}{\prod_{j}\left\langle\eta a_{i}\right\rangle}\left(\sum_{s_{0}=0}^{n-1} \frac{1}{\prod_{s=0, s \neq s_{0}}^{n-1}\left(s-s_{0}\right)} \frac{\prod_{j}\left(1+\tau s_{0} \frac{\left\langle\alpha b_{j}\right\rangle}{\left\langle\eta b_{j}\right\rangle}\right)}{\prod_{j}(1+}\right.
\end{aligned}
$$

We see that what we need to do is to expand the expression inside the parentheses as a series in $\tau$, keep only the terms up to order $\tau^{n-1}$. Although this expression has one auxiliary spinor $\eta$, the final result does 
not depend on it. Without writing out intermediate steps, we reach the following result:

$$
\begin{aligned}
& \frac{1}{\langle\eta \alpha\rangle^{n-1}} \frac{\prod_{i}\left\langle\eta a_{i}\right\rangle}{\prod_{j}\left\langle\eta b_{j}\right\rangle}\left(\sum_{s_{0}=0}^{n-1} \frac{s_{0}^{n-1}}{\prod_{s=0, s \neq s_{0}}^{n-1}\left(s-s_{0}\right)} \sum_{N_{1}+\sum_{j=1}^{k} m_{j}=n-1}\left(\prod_{j=1}^{k}(-)^{m_{j}}\left(\frac{\left\langle\alpha b_{j}\right\rangle}{\left\langle\eta b_{j}\right\rangle}\right)^{m_{j}}\right)\right. \\
& \left.\left(\sum_{1 \leq i_{1}<i_{2} \ldots<i_{N_{1}} \leq k+n-2} \prod_{q=1}^{N_{1}} \frac{\left\langle\alpha a_{i_{q}}\right\rangle}{\left\langle\eta a_{i_{q}}\right\rangle}\right)\right) .
\end{aligned}
$$

The third method is very similar to the second method. Upon noticing that

$$
\frac{d}{d \tau^{n-1}} \frac{1}{\langle\ell \eta-\tau s\rangle}=\frac{(n-1) !\langle\ell s\rangle^{n-1}}{\langle\ell \eta-\tau s\rangle^{n}},
$$

we can rewrite

$$
\begin{aligned}
\frac{1}{\langle\ell \eta\rangle^{n}} \frac{\prod_{i}\left\langle\ell a_{i}\right\rangle}{\prod_{j}\left\langle\ell b_{j}\right\rangle} & \rightarrow \frac{1}{\langle\ell \eta-\tau s\rangle^{n}} \frac{\prod_{i}\left\langle\ell a_{i}\right\rangle}{\prod_{j}\left\langle\ell b_{j}\right\rangle} \\
& =\frac{d}{d \tau^{n-1}}\left(\frac{1}{\langle\ell \eta-\tau s\rangle} \frac{1}{(n-1) !\langle\ell s\rangle^{n-1}} \frac{\prod_{i}\left\langle\ell a_{i}\right\rangle}{\prod_{j}\left\langle\ell b_{j}\right\rangle}\right) .
\end{aligned}
$$

Now we take the simple residue of $\langle\ell \eta-\tau s\rangle$. That is, we set $|\ell\rangle=|\eta-\tau s\rangle$, take the derivative, and finally find that the residue is

$$
\text { Residue }=\frac{d}{d \tau^{n-1}}\left(\frac{1}{(n-1) !\langle\eta s\rangle^{n-1}} \frac{\prod_{i}\left\langle(\eta-\tau s) a_{i}\right\rangle}{\prod_{j}\left\langle(\eta-\tau s) b_{j}\right\rangle}\right)_{\tau \rightarrow 0} .
$$

It should not be hard to check that (A.5) is the same as (A.7) if we identify $\alpha=s$. As a demonstration, let us check the case of a double pole:

$$
\begin{aligned}
& \left.\frac{1}{\langle\ell \eta-\tau s\rangle^{2}} \frac{\prod_{j=1}^{k}\left\langle\ell a_{j}\right\rangle}{\prod_{j=1}^{k}\left\langle\ell b_{j}\right\rangle} \rightarrow \frac{d}{d \tau}\left(\frac{1}{\langle\ell s\rangle\langle\ell \eta-\tau s\rangle} \frac{\prod_{j=1}^{k}\left\langle\ell a_{j}\right\rangle}{\prod_{j=1}^{k}\left\langle\ell b_{j}\right\rangle}\right)\right|_{\text {residue }} \\
= & \left.\frac{d}{d \tau}\left(\frac{1}{\langle\eta-\tau s s\rangle} \frac{\prod_{j=1}^{k}\left\langle\eta-\tau s a_{j}\right\rangle}{\prod_{j=1}^{k}\left\langle\eta-\tau s b_{j}\right\rangle}\right)\right|_{\tau \rightarrow 0} \\
= & \frac{1}{\langle\eta s\rangle} \frac{\prod_{j=1}^{k}\left\langle\eta a_{j}\right\rangle}{\prod_{j=1}^{k}\left\langle\eta b_{j}\right\rangle}\left(\sum_{j} \frac{\left\langle s b_{j}\right\rangle}{\left\langle\eta b_{j}\right\rangle}-\sum_{j} \frac{\left\langle s a_{j}\right\rangle}{\left\langle\eta a_{j}\right\rangle}\right) .
\end{aligned}
$$

This is exactly the same result given by the shifting technique.

Knowing how to evaluate the residue of multiple poles, we can discuss how to evaluate coefficients for triangles and bubbles given by (4.43) and (4.46). We use the third method. For example, for (4.43) we can shift $K \rightarrow K-\tau s$. Then using (A.6) we can transfer it to the residue of a simple pole. We can further simplify the sum of these two simple poles using the same technique discussed in our previous subsection for box coefficients. After that we can take the derivative and the $\tau \rightarrow 0$ limit by truncating the power series. 


\section{References}

[1] F. del Aguila and R. Pittau, JHEP 0407, 017 (2004) [arXiv:hep-ph/0404120].

[2] G. Ossola, C. G. Papadopoulos and R. Pittau, arXiv:hep-ph/0609007.

[3] R. K. Ellis, W. T. Giele and G. Zanderighi, JHEP 0605, 027 (2006) [arXiv:hep-ph/0602185].

R. K. Ellis, W. T. Giele and G. Zanderighi, Phys. Rev. D 73, 014027 (2006) [arXiv:hep-ph/0508308].

[4] C. Anastasiou and A. Lazopoulos, JHEP 0407, 046 (2004) [arXiv:hep-ph/0404258].

[5] R. Britto, F. Cachazo and B. Feng, Nucl. Phys. B 725, 275 (2005) [arXiv:hep-th/0412103].

[6] R. Britto, E. Buchbinder, F. Cachazo and B. Feng, Phys. Rev. D 72, 065012 (2005) [arXiv:hep-ph/0503132].

[7] R. Britto, B. Feng and P. Mastrolia, Phys. Rev. D 73, 105004 (2006) [arXiv:hep-ph/0602178].

[8] C. F. Berger, Z. Bern, L. J. Dixon, D. Forde and D. A. Kosower, Phys. Rev. D 74, 036009 (2006) [arXiv:hep-ph/0604195].

Z. Bern, L. J. Dixon and D. A. Kosower, Phys. Rev. D 73, 065013 (2006) [arXiv:hep-ph/0507005].

Z. Bern, L. J. Dixon and D. A. Kosower, Phys. Rev. D 71, 105013 (2005) [arXiv:hep-th/0501240].

[9] A. Brandhuber, S. McNamara, B. J. Spence and G. Travaglini, JHEP 0510, 011 (2005) [arXiv:hep-th/0506068].

[10] Z. Xiao, G. Yang and C. J. Zhu, Nucl. Phys. B 758, 1 (2006) [arXiv:hep-ph/0607015].

X. Su, Z. Xiao, G. Yang and C. J. Zhu, Nucl. Phys. B 758, 35 (2006) [arXiv:hep-ph/0607016].

Z. Xiao, G. Yang and C. J. Zhu, Nucl. Phys. B 758, 53 (2006) [arXiv:hep-ph/0607017].

T. Binoth, J. P. Guillet and G. Heinrich, arXiv:hep-ph/0609054.

[11] P. Mastrolia, arXiv:hep-th/0611091.

[12] Z. Bern, L. J. Dixon, D. C. Dunbar and D. A. Kosower, Nucl. Phys. B 425, 217 (1994)

[13] G. Passarino and M.J.G. Veltman, Nucl. Phys. B 160 (1979) 151;

Z. Bern, L. J. Dixon and D. A. Kosower, Phys. Lett. B 302, 299 (1993) [Erratum-ibid. B 318, 649 (1993)]

[arXiv:hep-ph/9212308].

O. V. Tarasov, Phys. Rev. D 54, 6479 (1996) [arXiv:hep-th/9606018].

[14] F. Cachazo, P. Svrcek and E. Witten, JHEP 0410, 077 (2004) [arXiv:hep-th/0409245].

[15] W. L. van Neerven, Nucl. Phys. B 268, 453 (1986).

[16] Z. Bern and A. G. Morgan, Nucl. Phys. B 467, 479 (1996) [arXiv:hep-ph/9511336].

[17] Z. Bern, L. J. Dixon and D. A. Kosower, Ann. Rev. Nucl. Part. Sci. 46, 109 (1996) [arXiv:hep-ph/9602280].

[18] Z. Bern, L. J. Dixon, D. C. Dunbar and D. A. Kosower, Phys. Lett. B 394, 105 (1997) [arXiv:hep-th/9611127].

[19] C. Anastasiou, R. Britto, B. Feng, Z. Kunszt and P. Mastrolia, arXiv:hep-ph/0609191.

[20] C. Anastasiou, R. Britto, B. Feng, Z. Kunszt and P. Mastrolia, arXiv:hep-ph/0612277. 
[21] C. Anastasiou, private communication.

[22] F. Cachazo and P. Svrcek, PoS RTN2005, 004 (2005) [arXiv:hep-th/0504194].

[23] L. J. Dixon, PoS HEP2005, 405 (2006) [arXiv:hep-ph/0512111].

[24] S. D. Badger, E. W. N. Glover, V. V. Khoze and P. Svrcek, JHEP 0507, 025 (2005) [arXiv:hep-th/0504159].

[25] Z. Bern, L. J. Dixon and D. A. Kosower, Nucl. Phys. B 513, 3 (1998) [arXiv:hep-ph/9708239].

[26] Z. Bern, V. Del Duca, L. J. Dixon and D. A. Kosower, Phys. Rev. D 71, 045006 (2005) [arXiv:hep-th/0410224].

[27] Z. Bern, L. J. Dixon and D. A. Kosower, Phys. Rev. D 72, 045014 (2005) [arXiv:hep-th/0412210].

[28] S. J. Bidder, N. E. J. Bjerrum-Bohr, D. C. Dunbar and W. B. Perkins, Phys. Lett. B 612, 75 (2005) [arXiv:hep-th/0502028]. 